\title{
Mesh Force Modelling and Parametric Studies for Compound Oscillatory Roller Reducer
}

\author{
Changxu Wei, Chaosheng Song ${ }^{*}$ (D), Caichao Zhu, Chengcheng Liang and Long Hu
}

\begin{abstract}
A compound oscillatory roller reducer (CORR) with a first-stage gear transmission and a second-stage oscillatory roller transmission is presented. The transmission principle of oscillatory roller transmission is introduced, and the tooth profile equation of the inner gear is derived. The analytical model of mesh force considering the installation errors and manufacturing errors is proposed. Then, parametric studies considering different errors on the mesh force are conducted. Results show that the design parameters are significant factors for mesh force. The mesh force is reduced by $17 \%$ as the eccentricity of disk cam increases from $2.5 \mathrm{~mm}$ to $4 \mathrm{~mm}$. When the radius of the movable roller increases from $7 \mathrm{~mm}$ to $20 \mathrm{~mm}$, the mesh force decreases by $8 \%$. As the radius of disk cam increases from $125 \mathrm{~mm}$ to $170 \mathrm{~mm}$, the mesh force is decreased by $26.5 \%$. For the impacts of errors, the mesh force has a noticeable fluctuation when these errors exist including the manufacturing error of disk cam, the installation error of disk cam and the manufacturing error of movable roller change. The prototype of the reducer is manufactured and preliminary run-in test proved the feasibility of the transmission principle.
\end{abstract}

Keywords: Compound oscillatory roller reducer, Kinematic principle, Mesh force modelling, Misalignments, Parametric study

\section{Introduction}

The compound oscillatory roller transmission is a new type of few teeth difference transmission with multitooth meshing. And it can be widely used in precision transmission for various servo drive systems, such as robots, $\mathrm{CNC}$ machine tools, and industrial automation. The compound oscillatory roller transmission is based on the oscillatory roller transmission with a single eccentric shaft structure. It not only overcomes the weakness of the harmonic reducer with low stiffness and large backlash, but also greatly solves the problem of large load and low life of the arm bearing in the RV reducer. At the same time, the accuracy, rigidity and power density of the reducer are improved and the manufacturing cost is

*Correspondence: chaoshengsong@cqu.edu.cn

The State Key Laboratory of Mechanical Transmissions, Chongqing University, Chongqing 400030, China lower. However, due to the special structure of CORR, the half of teeth participate in the meshing process and the mesh forces change with the meshing position. The force of movable rolling teeth is a complex over-determination problem. For such problems, it is impossible to calculate the force and distribution of the movable rolling teeth by using conventional methods, which cause great inconvenience to the design work. So, proposing an accurate method at calculating the force of movable rolling teeth is imperative.

In the existing studies, lots of researches were conducted for gear transmissions [1-5]. Sahoo et al. proposed a method to estimate the load shared by contact analysis of the multiple tooth pairs and the load distribution pattern in proportion to the tooth deformation was considered [6]. In the modeling, member considered the physical phenomena of the transmission error due to nonlinear elastic deformations in the 
micro-displacement region, and proposed a novel modeling and compensation approach for the angular transmission error in harmonic drive gearings [7]. Chen et al. [8] presented a method to design a tooth profile and proved the backlash assessment in harmonic drive to enable more teeth to participate in meshing. They also studied the influences of the double-circular-arc common-tangent tooth profile parameters on the distribution and the magnitude of the backlash. Dhaouadi et al. [9] put forward a mathematical model and its parameter identification scheme for harmonic drive gears with compliance and hysteresis. They used numerical simulations and experimental data to verify the modelling concept. Chen et al. [10] researched the deformation and stress distribution of harmonic drive in an assembly state and transmission state. They gave some suggestions for service life and high-performance design. They studied the important modes for the crankshaft of RV reducer using a finite element analysis method in the situation of free border and real border, then he obtained a frequency rang [11]. Zhang et al. [12] proposed a method can accurately calculate the manufacturing error of cycloidal gear for RV reducer. Yu et al. [13] used the finite element method to research the specific impact on the meshing force of manufacturing errors of the pins. Sun et al. [14] proposed a new one-stage cycloid-pin gear reducer. They presented the design and motion analysis of this reducer. Wu et al. [15] introduced the structure and operating principle for rolling movable teeth reducer. They studied the kinematics simulation and dynamic analysis by ADAMS software. Wang et al. [16] established the virtual prototype of the swing movable teeth reducer and achieved the dynamical simulation of the reducer by CAD/ CAE software. Liang et al. [17] put forward a method to build the finite element model for swing movable teeth reducer. They researched the natural frequencies and vibration modes of the reducer by using the Block Lanczos method. Song et al. [18] studied a novel pure rolling cycloid planetary gear reducer. They testified the validity and feasibility of the approach to the pure rolling cycloid gear reducer by kinematics simulation. Li et al. [19] analyzed the influence of configuration parameters on the contact ration for cam shock wave rolling movable teeth transmission, including the working teeth profile and curvature of teeth crest of center wheel. Yi et al. [20] introduced the structure and drive principle for rolling swing movable teeth transmission, then synthesized the theoretical and actual profile for the central wheel by methods of complex vector. Routh et al. [21] aimed at review on different aspects of harmonic drive gear to make the comparative study of harmonic drive gear over conventional gear, studying the different design aspects i.e., synthesis of tooth profiles, stress, strain, torque, load sharing and kinematics error, etc. Pham et al. [22] summarized high precision reducers (HPRs) for industrial robots. Then they discussed principle, characteristics, and three main performances of HPRs. Garcia et al. [23] proposed an assessment framework strongly conditioned by Human-RobotInteraction applications, and used it to review the performance of conventional and emerging robotic gearbox technologies. Li et al. [24] designed the wave generator profile for harmonic drive using a closed convex curve generated by the support function, and established an optimization model of the wave generator profile and a finite element assembly model. Jiang et al. [25] proposed a novel bidirectional conjugate design method, which is based on the combined conjugate calculation of the tooth profiles of flexspline and circular spline, to solve the uncertain problem of the conjugated convex circular-arc tooth profile of circular spline. They calculated the equivalent stress of the twotooth-difference oscillating-disc movable teeth transmission and studied the influence of the parameters of the movable tooth on the contact stress [26]. Yang et al. [27] analyzed the force of the movable tooth meshing pair of a two-step sine movable tooth transmission and proposed the formulas for calculating the transmission efficiency of the system. Cao et al. [28] introduced a micro-integrated movable tooth transmission system and deduced the static equations of any movable teeth at different meshing positions in micro-scale. $\mathrm{Li}$ et al. [29] proposed a swing output movable teeth cam mechanism, and analyzed its structure and transmission principle. Yi et al. [30] proposed a new model of five polynomial curves to achieve any tooth-difference pure rolling movable tooth transmission, and analyzed influence of the movable tooth axis radius on the properties of tooth profile.

In summary, the above studies analyzed the multitooth force situation without considering the error. However, the load distribution on each working wheel is very complicated in the multi-tooth meshing drive, and the load distribution is influenced by factors such as the deformation of the part, installation errors, manufacturing errors and meshing backlash. This paper mainly studies the influence of manufacturing errors and installation errors on mesh force for the compound oscillatory roller reducer. Firstly, the transmission principle of oscillatory roller transmission is introduced, and the tooth profile equation of the inner gear is derived. Then, the analytical model of the meshing force considering the installation errors and manufacturing errors is proposed. Finally, parametric studies considering various errors on the mesh force are conducted. 


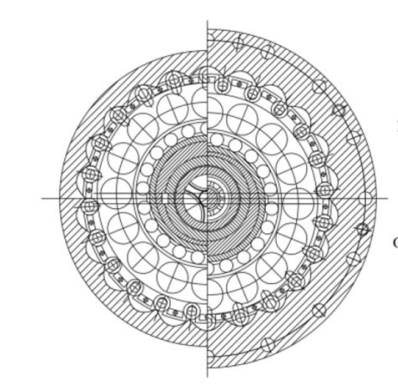

(a) Construction

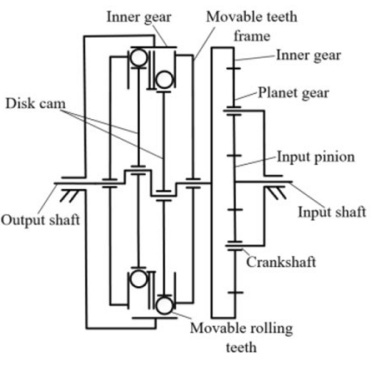

(b) Kinematic principle
Figure 1 Construction and kinematic principle of CORR

\section{Kinematic Principle and Construction of CORR}

The section introduces the structural characteristics and kinematic principle of the CORR. CORR is a twostage series structure with a first-stage gear transmission and a second-stage oscillatory roller transmission. The construction and kinematic principle are illuminated in Figure 1, respectively. In the first stage gear transmission, an input pinion meshes with three wheels mounted on the frame, and three wheels mesh with the inner gear mounted on the crankshaft. In the second stage oscillatory roller transmission, the crankshaft drives the disk cam for eccentric motion. To drive movable roller and provide a balanced load, the phase value is offset $180^{\circ}$ for two disk cams. The disk cam drives the movable rolling teeth to move radially in the groove of the movable teeth frame and engages with the inner gear. At the same time, the circumferential motion of movable rolling teeth is output by the movable teeth frame.

From the above analysis of the structural characteristics and kinematic principle of CORR, the following characteristics can be obtained.

(1) Compact structure: Comparing with the RV reducer, CORR has no output mechanisms and is directly output by the movable teeth frame. In addition, the disk cam, movable rolling teeth, and inner gear are coaxially arranged, and the axial size of CORR is small, thereby reducing the volume and weight.

(2) Strong carrying capacity: All the movable rolling teeth are in contact with the inner gear, and at most half of the movable rolling teeth are engaged at the same time, so the carrying capacity is strong. Multitooth meshing also makes movable rolling teeth withstand more shock loads.

(3) Large transmission ratio: CORR is a planetary gear transmission with less tooth difference. The transmission ratio of oscillatory roller transmission can reach 8-60, and the transmission ratio of the twostage can reach 64-3600.

In the design process of the CORR, the most complicated part is the tooth profile of the inner gear. Therefore, the other parameters of the CORR are generally selected first, and the tooth profile equation of the inner gear is derived based on the principle of constant velocity conjugate and envelope.

As shown in Figure 2(a), the equivalent low-low mechanism of oscillatory roller transmission's meshing pair is a two DOFs five-bar linkage composed of crank 1, copular link 2, slider 3 , guide link 4 and frame 5 , in which the crank 1 and guide link 4 rotate around the same axis. The parameters $L_{1}$ and $L_{2}$ are denoted the lengths of the crank 1 and copular link 4 . The symbols $\theta_{1}$ and $\theta_{2}$ are the rotational angles of the crank 1 and guide link 4 . The displacement of point $H$ along the guide link can be written as $C_{H}=f\left(L_{1}, L_{2}\right.$, $\left.\theta_{1}, \theta_{2}\right)$. Because the ratio of CORR is fixed, $i_{1,2}=\mathrm{d} \theta_{1} /$ $\mathrm{d} \theta_{2}=\omega_{1} / \omega_{2}=$ const. Therefore, the track $C-C$ of point $H$ is obtained when the parameters $\theta_{1}$ and $\theta_{2}$ are given. The rotation angle $\theta_{2}$ of guide link must follow the rule according to the invariable speed rate $i_{1,2}$.

Based on Figure 2(a), the conjugate tooth profile of the inner gear is obtained by the envelope principle as shown in Figure 2(b). Where the eccentricity of the disk cam is $e=L_{1}$, the radius of the disk cam is $R_{j}$, the radius of the movable roller is $r$, and $R_{j}+r=L_{2}$. Let $C^{\prime}-C^{\prime}$ represent the outer equidistant curve of $C$ - $C$ relative to the roller radius $r$ based on the envelope principle, then the equations of curves $C-C$ and $C^{\prime}-C^{\prime}$ (the theoretic and work profiles of inner gear) as follows

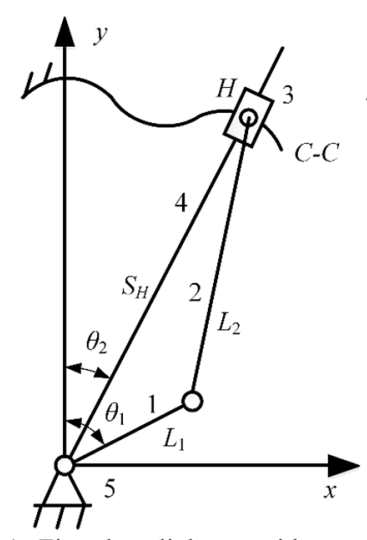

(a) Five bar linkage with two DOFs combinatorial linkage

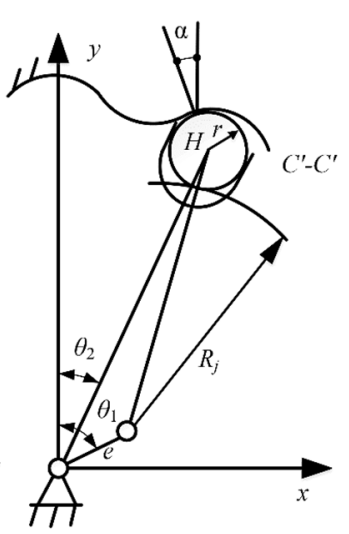

(b) Cam-link-roller
Figure 2 Schematic diagram of oscillatory roller transmission 


$$
\begin{aligned}
& \left\{\begin{array}{l}
x_{H}=e\left(\cos z \theta_{2}+\sqrt{\left(R_{j}+r\right)^{2}-\sin ^{2} z \theta_{2}}\right) \sin \theta_{2}, \\
y_{H}=e\left(\cos z \theta_{2}+\sqrt{\left(R_{j}+r\right)^{2}-\sin ^{2} z \theta_{2}}\right) \cos \theta_{2},
\end{array}\right. \\
& \left\{\begin{array}{l}
x=e\left(\cos z \theta_{2}+\sqrt{\left(R_{j}+r\right)^{2}-\sin ^{2} z \theta_{2}}\right) \sin \theta_{2}+r \sin \alpha, \\
y=e\left(\cos z \theta_{2}+\sqrt{\left(R_{j}+r\right)^{2}-\sin ^{2} z \theta_{2}}\right) \cos \theta_{2}+r \cos \alpha,
\end{array}\right.
\end{aligned}
$$

where $z$ is the tooth number of fixed inner gear, $\alpha$ is the angle between $y$ axis and the normal line of curve $C^{\prime}-C^{\prime}$ at the mesh point. $\alpha$ can be calculated by Eq. (3). tortions of the elements of CORR are not considered.

(2) Because of the small mass of the movable roller, the influence of its inertial force is ignored.

(3) Ignore the friction forces of movable roller.

The oscillatory roller transmission is equivalent to the parallel cam-guide link-roller linkages as shown in Figure 3. The entire transmission achieves motion and power transmission through many identical parallel combinations. Therefore, the force analysis of the meshing pair of the movable roller $i$ can be performed, and a model based on this for meshing force can be established [31].

$$
\alpha=\arctan \left(-\frac{z \sin z \theta_{2} \sin \theta_{2}+\sqrt{\left(R_{j}+r\right)^{2}-\sin ^{2} z \theta_{2}} \cdot \cos \theta_{2}}{z \sin z \theta_{2} \cos \theta_{2}-\sqrt{\left(R_{j}+r\right)^{2}-\sin ^{2} z \theta_{2}} \cdot \sin \theta_{2}}\right) .
$$

The design parameters of CORR are shown in Table 1.

\section{Mesh Force Modelling for CORR with Error}

In this section, a mesh force model considering errors is established based on the structural characteristics and kinematic principle of the CORR. In order to analyze the mesh force for simple and convenient, there are three assumptions as shown in the following.

Recently, various models of the human upper-limb anatomy have been derived. The biomechanical models of the arm that stand for precise anatomical models including muscles, tendons and bones are too complex to be utilized in mechanical design of an anthropomorphic robot arm. From the view of the mechanism, we should set up a more practicable model for easy and effective realization.

(1) The contact distortions between the movable roller and the disk cam, the movable teeth frame, and the inner gear are small and stretchable, and other dis-

Table 1 Design parameters of CORR

\begin{tabular}{lll}
\hline Parameter & Symbol & Value \\
\hline Teeth number of the inner gear & $Z$ & 25 \\
Transmission ratio & $i$ & 26 \\
The radius of the disk cam $(\mathrm{mm})$ & $R_{j}$ & 135 \\
The radius of the movable roller $(\mathrm{mm})$ & $r$ & 8 \\
The length of the movable roller $(\mathrm{mm})$ & $I_{H}$ & 12 \\
The eccentricity of the disk cam $(\mathrm{mm})$ & $e$ & 3.5 \\
The Young's modulus $(\mathrm{MPa})$ & $E$ & $2.06 \times 10^{5}$ \\
The Poisson's ratio & $\mu$ & 0.3 \\
\hline
\end{tabular}

$H_{i}$ is the center point of the movable teeth. $F_{j i}$ is the force between the disk cam and the movable roller. $F_{s i}$ and $F_{n i}$ are denoted the forces of the movable teeth frame and the inner gear acting on the movable roller respectively. From Figure 3, the force equilibrium of the movable teeth can be written as

$$
\begin{aligned}
F_{s i} / \sin \left(\gamma_{i}+\theta_{i}+\alpha_{i}\right) & =F_{n i} / \sin \left(\pi / 2-\gamma_{i}\right) \\
& =F_{j i} / \sin \left(\pi / 2-\theta_{i}-\alpha_{i}\right),
\end{aligned}
$$

where $\alpha_{i}$ is the angle between $y$ axis and the normal vector of the inner gear profile at the mesh point; $\theta_{i}$ is denoted the angle between $y$ axis and the connecting line of the movable teeth frame axis and the center of the movable teeth.

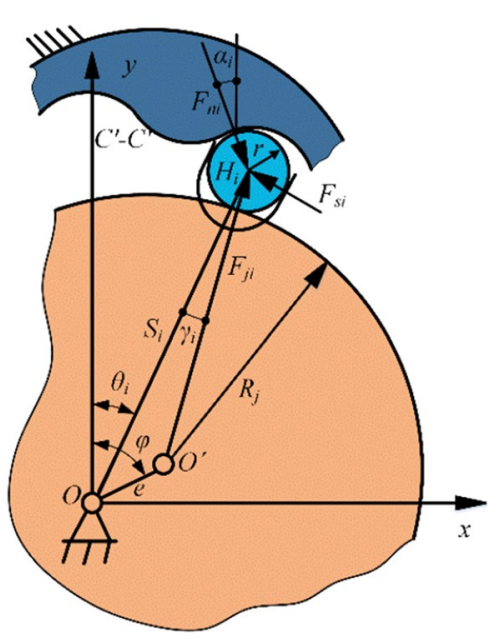

Figure 3 Force analysis of cam-guild link-roller combinatorial linkage mechanical model 


$$
\theta_{i}=\theta+(i-1) \Delta \theta
$$

In Eq. (5), $\Delta \theta=2 \pi /(z+1)$ denotes the central angle between two adjacent movable teeth, $\theta$ is the initial output angle of the movable teeth frame.

The parameter $\gamma_{i}$ is the angle between two lines, one is the connecting line between the movable teeth frame axis and the center of movable teeth and the other is the connecting line between the centers of the disk cam and movable teeth. According to Figure 3, $\gamma_{i}$ can be written as follow

$$
\gamma_{i}=\cos ^{-1}\left(\left(s_{i}^{2}+R_{j}^{2}-e^{2}\right) / 2 s_{i} R_{j}\right),
$$

where $s_{i}$ is the distance between the movable teeth frame axis and the center of movable teeth.

$$
s_{i}=e \cos \left(z \theta_{i}\right)+\sqrt{\left(R_{j}^{2}-e^{2} \sin ^{2}\left(z \theta_{i}\right)\right)},
$$

The moments acting on the two disk cams can be calculated. When CORR works stably, the input torque must be equal to the output torque. If one disk cam is selected, considering the force asymmetry of the two disk cams, a coefficient 0.55 is multiplied in the equation. Half of the movable rolling teeth endure meshing force, and the disk cam only receives the forces of movable teeth. The moment equilibrium equation of one disk cam can be calculated as follow

$$
0.55 M_{0}=\sum_{i=1}^{(z+1) / 2} F_{j i} e \sin \left(\gamma_{i}-\theta_{i}+\varphi\right),
$$

where $M_{0}$ is the input moment by the crankshafts, $\phi$ denotes the input angle of the disk cam corresponding to the initial output angle of the movable teeth frame. The expression $\varphi=(z+1) \theta$ can be obtained.

When the input moment of the small input gear is given, the input moment $M_{0}$ can be calculated by using the same method as RV reducer, because the first stage of CORR is the same as RV reducer.

The force translation is through the stretch contact distortion between the disk cam, movable teeth, and movable teeth frame according to the structure of CORR. Suppose the distortion is linear and small. It can be described approximately by the small area with width $L_{1}$ and length $L_{2}$ shown in Figure 4 , and $L_{1}$ can be determined through Hertz's formula.

$$
L_{1}=\sqrt{\frac{4 F}{\pi L_{2}}\left[\lambda\left(\frac{1-\mu_{1}^{2}}{E_{1}}+\frac{1-\mu_{2}^{2}}{E_{2}}\right)\right]},
$$

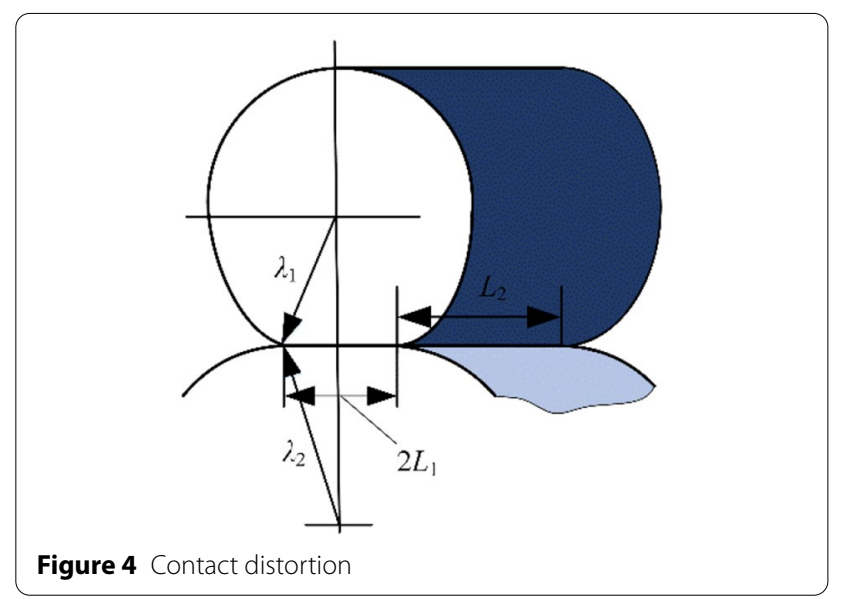

where $F$ is denoted the contact force between the two contact elements. $\mu_{1}, \mu_{2}$ and $E_{1}, E_{2}$ are Poisson's ratio and Young's modulus, respectively.

$$
\frac{1}{\lambda}=\frac{1}{\lambda_{1}} \pm \frac{1}{\lambda_{2}}
$$

where $\lambda_{1}$ is the radius of the movable roller, and $\lambda_{2}$ denotes the radius of the disk cam and the inner gear, "+" is used for convexity with convexity contact, "-" is used for convexity with concave contact. The contact surface of the movable teeth frame is flat, and the radius of curvature at the mesh point of contact with the movable roller is infinite. So, the equation can be changed to $1 / \lambda=1 / \lambda_{1}$.

According to Figure 4, the contact distortion of two elements can be written as

$$
\delta=\left(\lambda_{1} \pm \lambda_{2}\right)-\left(\sqrt{\lambda_{2}^{2}-L_{2}^{2}} \pm \sqrt{\lambda_{1}^{2}-L_{1}^{2}}\right)
$$

In the above equation, "+" is used when they are convexity with convexity contact, and "-" is used when they are convexity with concave contact. For plane with convexity contact, the contact distortion can be expressed by Eq. (12).

$$
\delta=\lambda_{1}-\sqrt{\lambda_{1}^{2}-L_{1}^{2}}
$$

From Figure 5(a), due to the output shaft is fixed, the parameters $\delta_{j i}, \delta_{s i}$ and $\delta_{n i}$ are the contact distortions between the movable teeth and the disk cam, movable teeth frame, and inner gear, respectively.

The movable roller will deviate the original position because of those above contact distortions. The excursion will cause a small displacement $\varepsilon_{i}$ of the disk cam along the direction of distortion $\delta_{j i}$ as shown in Figure 5(b). 


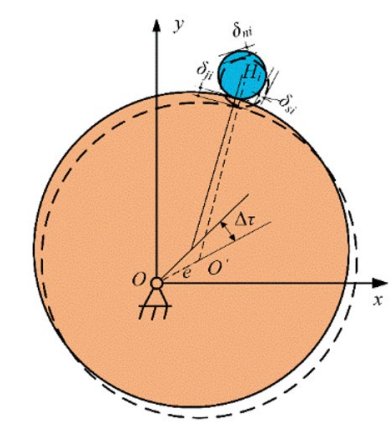

(a) Distortion congruity

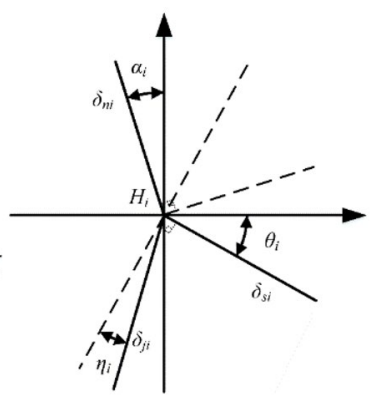

(b) Distortion transition
Figure 5 Condition of compatibility of movable teeth

$$
\varepsilon_{i}=\delta_{j i}+\delta_{s i}^{\prime}+\delta_{n i}^{\prime},
$$

where $\delta_{s i}^{\prime}, \delta_{n i}^{\prime}$ denote the incidental displacements of $\delta_{s i}$ and $\delta_{n i}$ respectively in the direction of $\delta_{j i}$.

$$
\begin{aligned}
& \delta_{s i}^{\prime}=\delta_{s i} \tan \theta_{i} \cos \gamma_{i}, \\
& \delta_{n i}^{\prime}=\delta_{n i} \cos \gamma_{i} / \cos \left(\theta_{i}-\alpha_{i}\right) .
\end{aligned}
$$

According to Figures 2 and 5, the displacement $\varepsilon_{i}$ can be compensated by a small rotation of the disk cam with a small angle $\Delta \tau$.

$$
\varepsilon_{i}=\Delta \tau\left|O O_{1}\right|=e \Delta \tau \sin \gamma_{i} .
$$

According to Eqs. (4)-(16), the meshing force analysis model of CORR is established. The meshing force is calculated through the following steps as shown in Figure 6.

Step 1. According to the given geometrical and operating state parameters, the relative parameters can be calculated, such as $\alpha_{i}, \gamma_{i}$ and the curvature radiuses.

Step 2. Obtain the expressions of $F_{s i}$ and $F_{n i}$, in which $F_{j i}$ is the variable, by using Eq. (4).

Step 3. To obtain the expressions of contact distortions $\delta_{j i}, \delta_{s i}$ and $\delta_{n i}$, we can substitute $F_{j i}, F_{s i}$ and $F_{n i}$ into Eq. (9), then substitute Eqs. (9) and (10) into Eqs. (11) and (12). In these expressions, $F_{j i}$ is the variable.

Step 4. To obtain the expressions of $\delta_{s i}^{\prime}$ and $\delta_{n i}^{\prime}$, in which $F_{j i}$ is the variable, substitute $\delta_{s i}$ and $\delta_{n i}$ into Eqs. (14) and (15).

Step 5. Give the initial value of $\Delta \tau$, and use Eq. (16) to determine $\varepsilon_{i}$.

Step 6. Substitute $\varepsilon_{i}, \delta_{j i}, \delta_{s i}^{\prime}$ and $\delta_{n i}^{\prime}$ into Eq. (13) to calculate $F_{j i}$.

Step 7. Use $\Delta \sigma=\left|\sum_{i=1}^{(z+1) / 2} F_{j i} e \sin \left(\gamma_{i}-\theta_{i}+\varphi\right)-0.55 M_{0}\right|$ as the iterative controlling variable. If $\Delta \sigma$ satisfy preci-

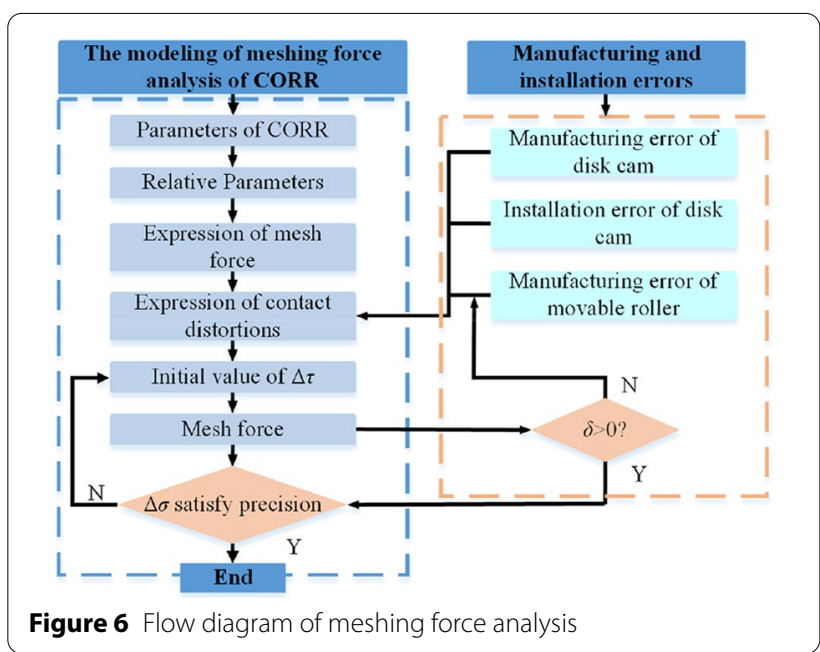

sion, stop the iterative calculation and calculate the meshing forces $F_{s i}$ and $F_{n i}$ according to the obtained $F_{j i}$. If not, make tiny adjustments to $\Delta \sigma$ and repeat step 5 to step 7 .

Geometric errors generally refer to the manufacturing and installation errors of key components in the oscillatory roller transmission. In this paper, the installation error and manufacturing error of the disk cam and the manufacturing error of the movable roller are mainly considered.

\subsection{Model of Mesh Force Considering Manufacturing Error of Disk Cam}

In actual situations, there are manufacturing errors of the disk cam, which will affect the mesh force. As shown in Figure 7 , there are manufacturing errors before the motion of Figure 5 . The radius of the disk cam and the contact normal distortions between the movable roller and the disk cam can be shown as Eqs. (17)-(19).

$$
\begin{aligned}
& R_{j_{-} \Delta R_{j}}=R_{j} \pm \Delta R_{j}, \\
& \delta_{j i \_\Delta R_{j}}=\delta_{j i} \pm \Delta \delta_{j i}=\delta_{j i} \pm \Delta R_{j}, \\
& \delta_{n i_{-} \Delta R_{j}}=\delta_{n i} \pm \Delta \delta_{n i}=\delta_{j i} \pm \Delta R_{j} \cos \alpha_{i},
\end{aligned}
$$

where $\Delta R_{j}$ is the manufacturing error of the disk cam. The symbol "+" is used for positive errors and "-" is used for negative errors.

Eqs. (17)-(19) are brought back to Eqs. (4)-(16). Then the mesh force is calculated through the above steps. But Step 7 should be added: if $\delta_{j i \_} \Delta R j>0$, the process continues 


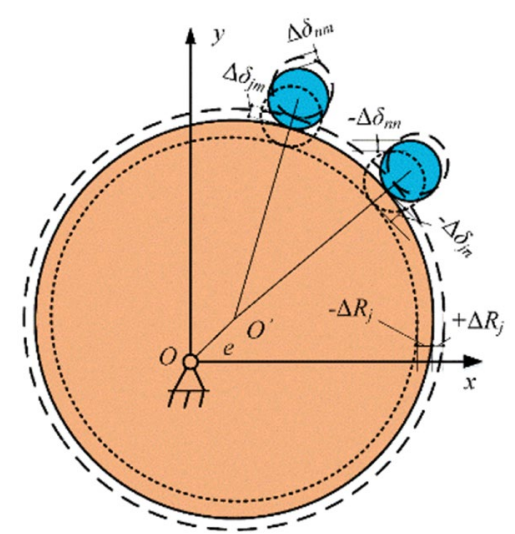

Figure 7 Distortion with manufacturing error of disk cam

calculating. If not, there is no distortion between the movable roller and the disk cam, then the mesh force $F_{j i}=0$.

\subsection{Model of Mesh Force Considering Installation Error of Disk Cam}

As shown in Figure 8, there are installation errors before the motion of Figure 5. The eccentricity of the disk cam and the contact normal distortions between the movable roller and the disk cam become Eqs. (20)-(23).

$$
\begin{aligned}
& e_{\Delta e}=e \pm \Delta e, \\
& \delta_{j i_{-} \Delta e}=\delta_{j i} \pm \Delta \delta_{j i}=\delta_{j i} \pm \Delta e \cos \theta_{i}, \\
& \delta_{s i \_\Delta e}=\delta_{s i} \pm \Delta \delta_{s i}=\delta_{s i} \pm \Delta e \tan \gamma_{i}, \\
& \delta_{n i_{-} \Delta e}=\delta_{n i} \pm \Delta \delta_{n i}=\delta_{n i} \pm \Delta e \cos \left(\theta_{i}-\alpha_{i}\right),
\end{aligned}
$$

where $\Delta e$ is the installation errors of the disk cam. "+" is used for positive errors and "-" is used for negative errors.

Eqs. (20)-(23) are brought back to the original formulas, and the other formulas are unchanged. Then calculate the mesh force through the above steps. However, Step 7 should be added: if $\delta_{j i} \Delta e>0$, the process continues calculating. If not, there is no distortion between the movable roller and disk cam, then the mesh force $F_{j i}=0$.

\subsection{Model of Mesh Force Considering Manufacturing Error of Movable Roller}

During the processing of a movable roller, there are inevitable manufacturing errors, which will affect the mesh force. As shown in Figure 9, the manufacturing errors can be expressed as

$$
\begin{aligned}
& r_{\Delta r}=r \pm \Delta r, \\
& \delta_{j i \_\Delta r}=\delta_{j i} \pm \Delta \delta_{j i}=\delta_{j i} \pm \Delta r, \\
& \delta_{s i_{-} \Delta r}=\delta_{s i} \pm \Delta \delta_{s i}=\delta_{s i} \pm \Delta r, \\
& \delta_{n i_{-} \Delta r}=\delta_{n i} \pm \Delta \delta_{n i}=\delta_{n i} \pm \Delta r,
\end{aligned}
$$

where $\Delta r$ is the manufacturing errors of the movable roller. "+" is used for positive errors, and "-" is used for negative errors.

Eqs. (24)-(27) are brought back to Eqs. (4)-(16). Then calculate the mesh force through the above steps. However, Step 7 should be changed: if $\delta_{j i \_}>0$, the process continues calculating. If not, there is no distortion between the movable teeth and the disk cam, movable teeth frame and inner gear, then the mesh force $F_{j i}=0$, $F_{s i}=0, F_{n i}=0$.

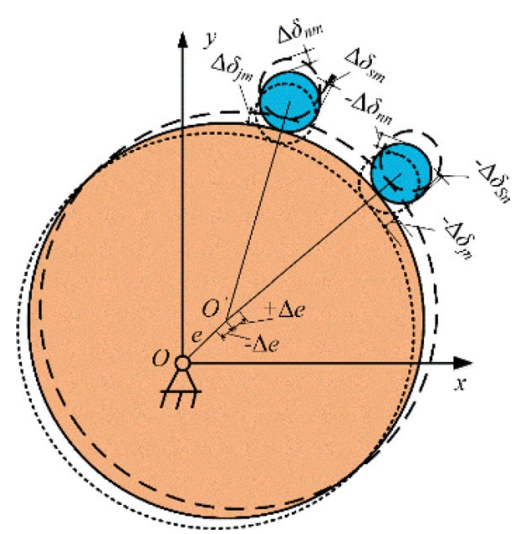

Figure 8 Distortion with installation error of disk cam

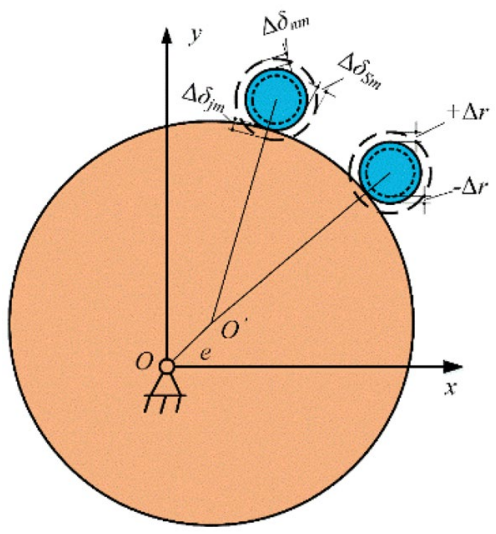

Figure 9 Distortion with manufacturing error of movable roller 


\section{Impacts of Parameters and Error on Mesh Force}

This section analyzes the influence of changes in structural parameters, installation errors and manufacturing errors on the meshing force. As shown in Figure 10, it's the working process of a movable roller in the meshing pair. The left side of the inner gear is the working tooth profile and the right side is the non-working tooth profile. The movable roller 1 exits the mesh motion from the end of the previous motion cycle and begins a new cycle. The work cycle includes two parts. Firstly, the movable roller occupies a position from 1 to 3 in sequence along the working tooth profile of the inner gear. Then, the movable roller enters the empty return stroke from position 3 and ends at position 5 .

The symbols $F_{j}, F_{s}$ and $F_{n}$ are the forces of the disk cam, the movable tooth frame and the inner gear acting on the movable roller, respectively. At first, the impacts of design parameters on mesh force are discussed. The mesh force varies with the change of eccentricity for the disk cam. The effects of the radius of the movable roller and the disk cam on mesh force are shown in Figure 11.

It is obvious that the increase of the eccentricity of disk cam makes the disk cam closer to the movable roller, which is easy to deform with the meshing process. There is a strong impact of the eccentricity of disk cam on mesh force. When the eccentricity of disk cam increases from $2.5 \mathrm{~mm}$ to $4 \mathrm{~mm}$, the maximum value of mesh force $F_{j}$ and $F_{n}$ are both changed and the maximum value is about $17 \%$ (from $4100 \mathrm{~N}$ to $2100 \mathrm{~N}$ ). The mean value of mesh force reduces by $16.7 \%$ (from $2430 \mathrm{~N}$ to $2050 \mathrm{~N}$ ). However, the peak to peak value and the mean value of mesh force $F_{s}$ are changed by a maximum of less than $1 \%$ and the changes are negligible.

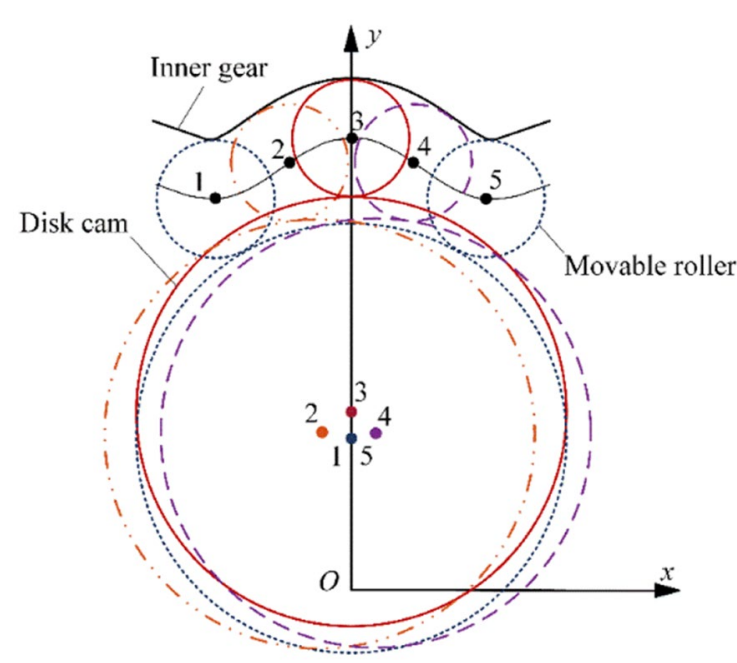

Figure $\mathbf{1 0}$ Geometrical model of meshing state
When the design parameter jumps from the eccentricity of the disk cam to the radius of the movable roller, the changing trends of mesh force are shown in Figure 12. The result shows that the radius of a movable roller has little effect on the mesh force. The mean value of mesh force $F_{j}$ and $F_{n}$ reduce by less $1 \%\left(F_{j}\right.$ reduces from 1735 $\mathrm{N}$ to $1724 \mathrm{~N}$ and $F_{n}$ reduces from $1770 \mathrm{~N}$ to $\left.1757 \mathrm{~N}\right)$. The mean value of mesh force $F_{s}$ reduces by $8 \%$ (from $229 \mathrm{~N}$ to $221 \mathrm{~N}$ ). The peak value of mesh force $F_{j}$ and $F_{n}$ both have few changes (from $2990 \mathrm{~N}$ to $2970 \mathrm{~N}$ ). The peak to peak value of mesh force $F_{s}$ has a little change of $7.8 \%$ (from $1140 \mathrm{~N}$ to $1050 \mathrm{~N}$ ). These laws of changes show that the mesh force is smooth when the radius of the movable roller changes.

Also, the results in Figure 13 show that the influence of the radius of disk cam is smaller compared with the eccentricity of disk cam. The mean value of mesh force $F_{j}$ and $F_{n}$ both reduce by approximate $1 \%\left(F_{j}\right.$ reduces from $1733 \mathrm{~N}$ to $1710 \mathrm{~N}$ and $F_{n}$ reduces from $1775 \mathrm{~N}$ to 1746 $\mathrm{N})$. The mean value of mesh force $F_{s}$ has a huge change of $26.5 \%$ (from $245 \mathrm{~N}$ to $180 \mathrm{~N}$ ). The peak value of mesh force $F_{j}$ and $F_{n}$ both reduce by approximately $1.5 \%\left(F_{j}\right.$ reduces from $2999 \mathrm{~N}$ to $2952 \mathrm{~N}$, and $F_{n}$ reduces from $3003 \mathrm{~N}$ to $2955 \mathrm{~N}$ ). The peak to peak value of mesh force $F_{s}$ reduces by $22.5 \%$ (from $1200 \mathrm{~N}$ to $920 \mathrm{~N}$ ).

From Figure 14, the mean mesh force $F_{j}, F_{n}$ and $F_{s}$ fall but change a little when the manufacturing errors of disk cam increase from $-0.02 \mathrm{~mm}$ to $0.02 \mathrm{~mm}$. The mean value of mesh force $F_{j}$ and $F_{n}$ both reduce by $2 \%\left(F_{j}\right.$ reduces from $1745 \mathrm{~N}$ to $1710 \mathrm{~N}$ and $F_{n}$ reduces from 1784 $\mathrm{N}$ to $1749 \mathrm{~N}$ ). The mean value of mesh force $F_{s}$ has a little change of $1.7 \%$ (from $229 \mathrm{~N}$ to $225 \mathrm{~N}$ ). However, the peak value of mesh force $F_{j}$ and $F_{n}$ increase by $2 \%\left(F_{j}\right.$ reduces from $2960 \mathrm{~N}$ to $3230 \mathrm{~N}$, and $F_{n}$ reduces from $2958 \mathrm{~N}$ to $3022 \mathrm{~N}$ ). The peak to peak value of mesh force $F_{s}$ has a change of $2.5 \%$ (from $1120 \mathrm{~N}$ to $1139 \mathrm{~N}$ ).

From Figure 15, the result shows that the installation error of disk cam has a huge effect on the mesh force. The mean value of mesh force $F_{j}$ and $F_{n}$ have a fluctuating change, but not a huge change. The mean value of mesh force $F_{s}$ reduces by $25 \%$ (from $239 \mathrm{~N}$ to $181 \mathrm{~N}$ ). The peak value of mesh force $F_{j}$ and $F_{n}$ rise smoothly as the installation error of disk cam grows to $0.01 \mathrm{~mm}$ and rises rapidly after $0.01 \mathrm{~mm}$. The peak to peak value of mesh force $F_{s}$ declines by $14.7 \%$ (from $1150 \mathrm{~N}$ to $960 \mathrm{~N}$ ).

From Figure 16, the result shows that the influence of the manufacturing error of movable roller is stronger compared with the other two errors. The mean value of mesh force $F_{j}$ and $F_{n}$ reduce by $6.7 \%$ and $6.5 \%\left(F_{j}\right.$ reduces from $1800 \mathrm{~N}$ to $1680 \mathrm{~N}$, and $F_{n}$ reduces from $1840 \mathrm{~N}$ to $1720 \mathrm{~N}$ ). The mean value of mesh force $F_{s}$ decrease by $14.6 \%$ (from $246 \mathrm{~N}$ to $210 \mathrm{~N}$ ). The peak value has the opposite trend. The peak value of mesh force $F_{j}$ and $F_{n}$ 


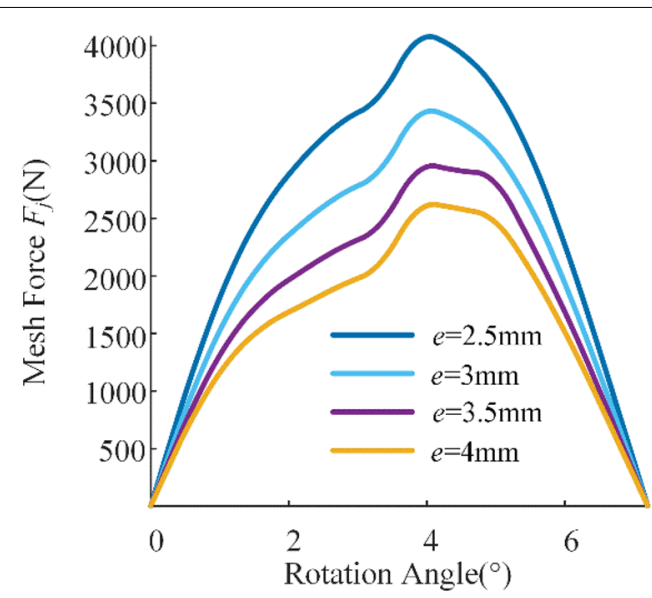

(a) Angle domain diagram

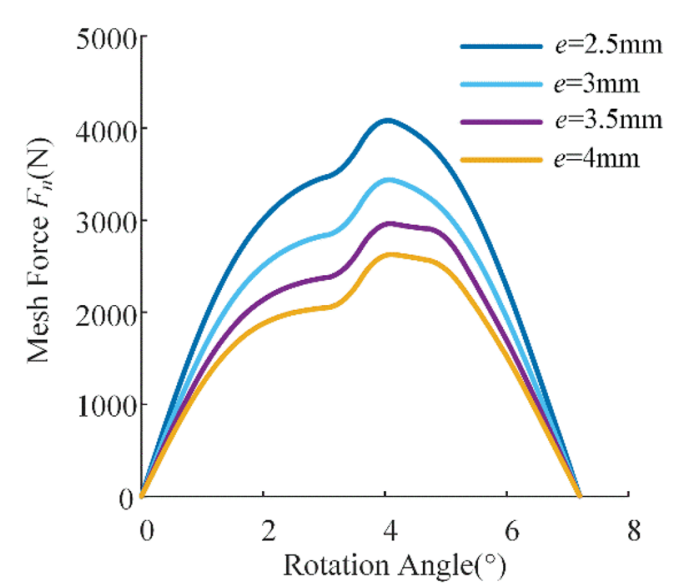

(c) Angle domain diagram

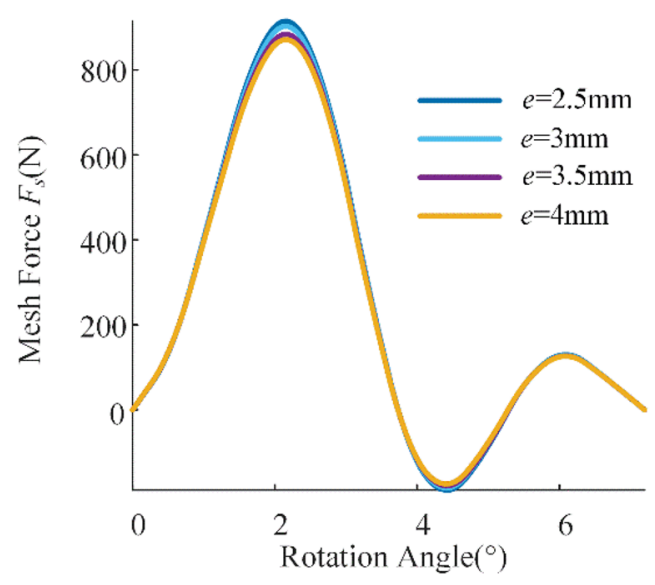

(e) Angle domain diagram

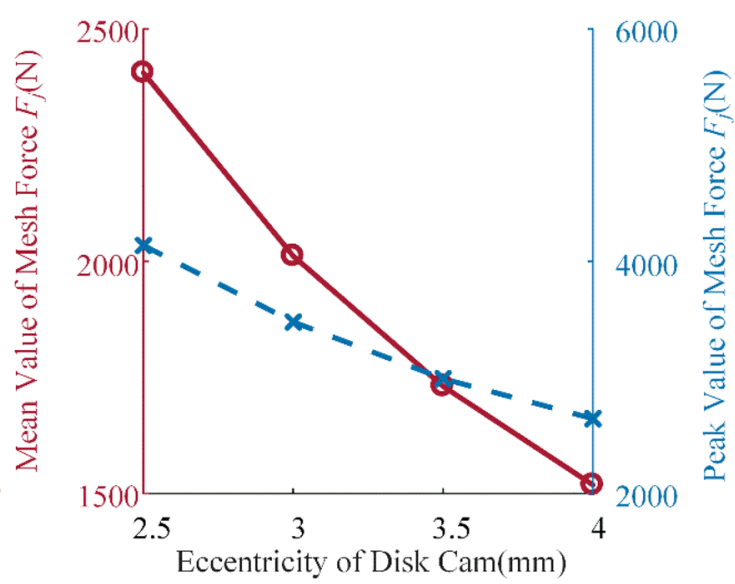

(b) Mean value and peak value

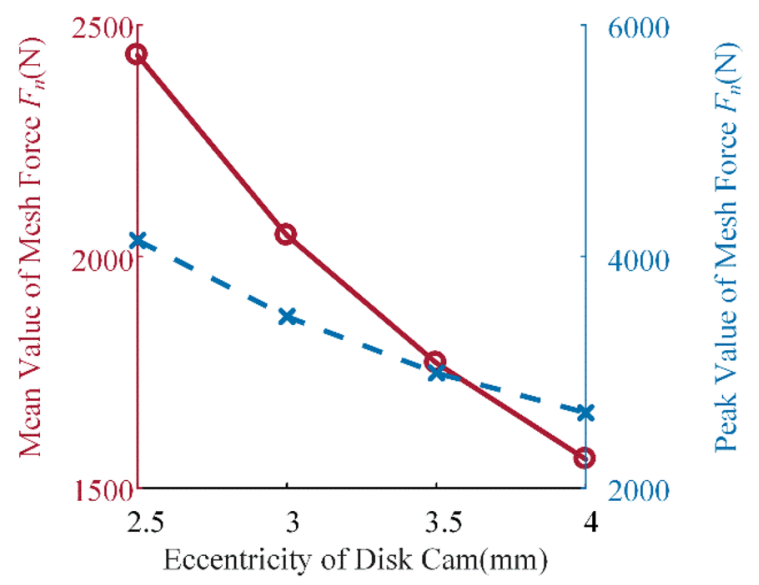

(d) Mean value and peak value

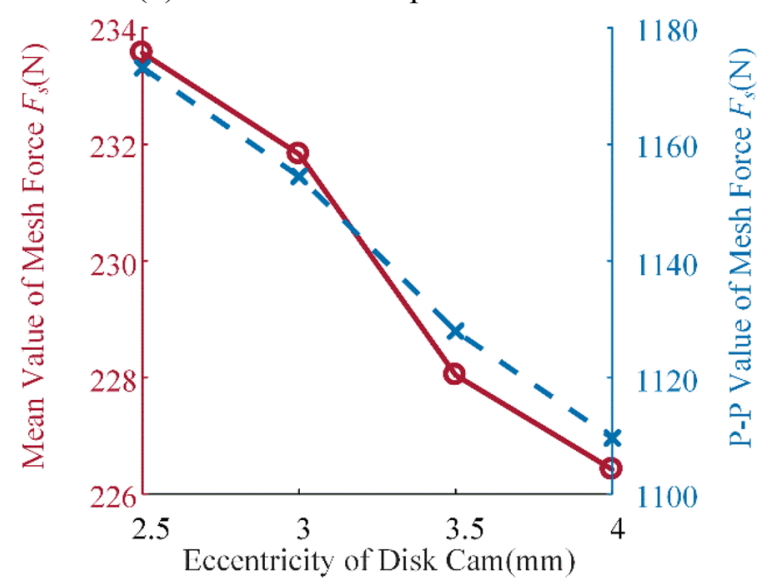

(f) Mean value and peak-peak value

Figure 11 Impacts of eccentricity of disk cam on mesh force 


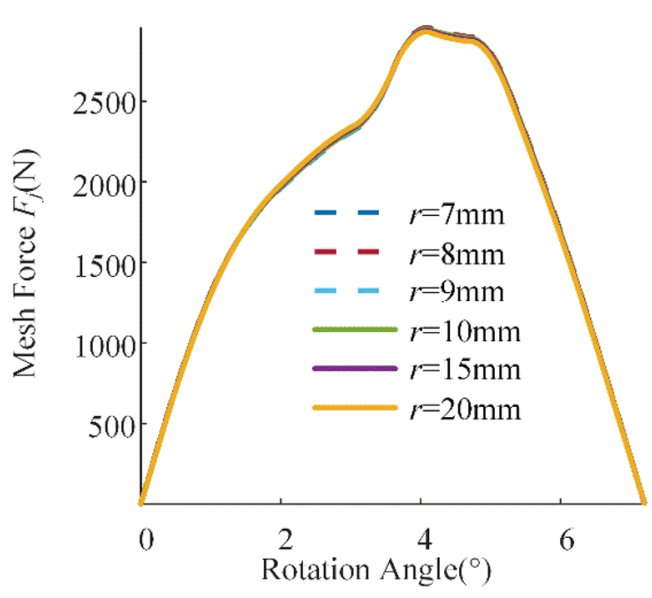

(a) Angle domain diagram

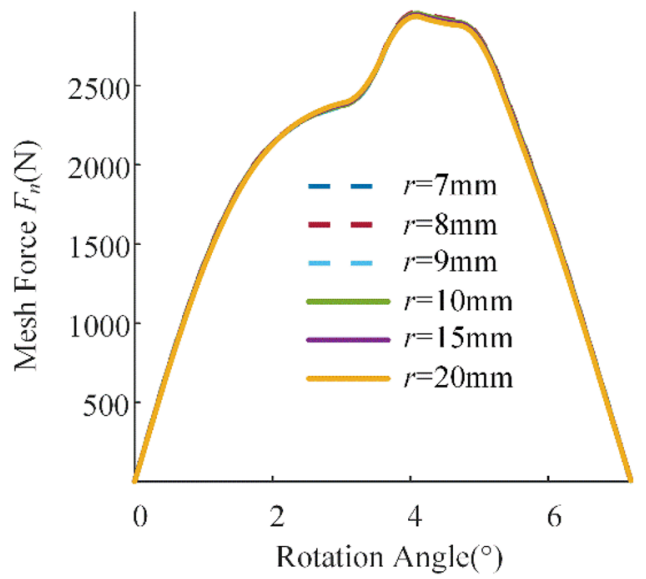

(c) Angle domain diagram

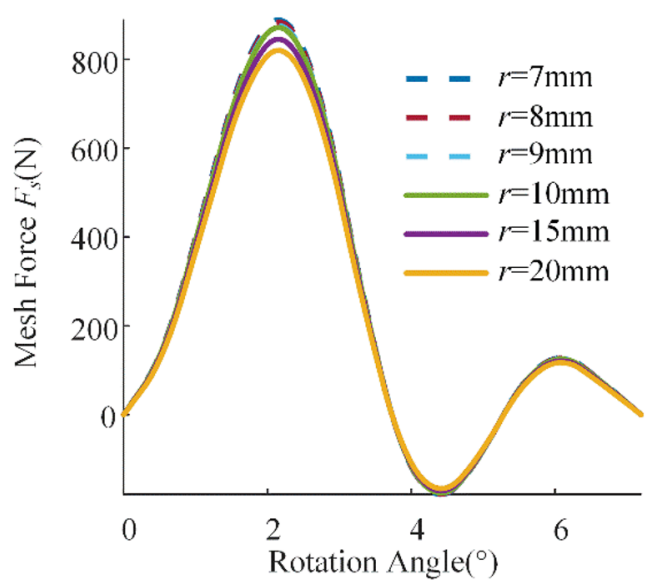

(e) Angle domain diagram

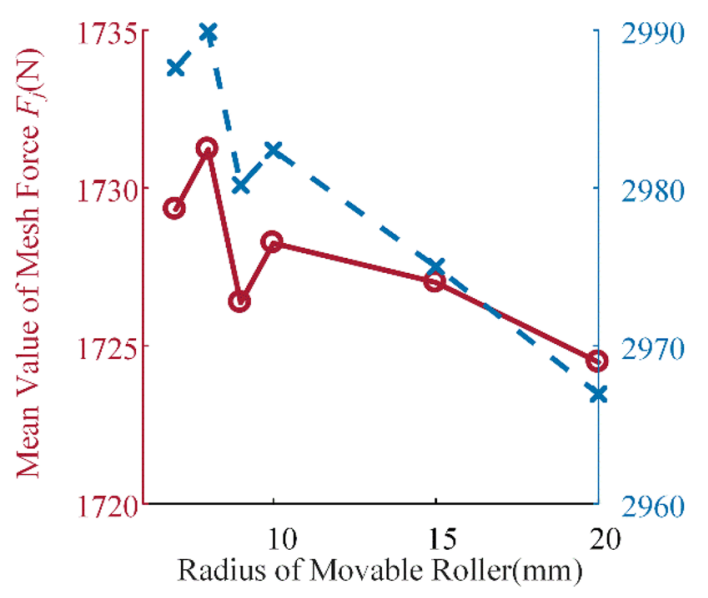

$z$
0
0
0
0
0
0
0
0
0
0
0
0
0
0
0
0

(b) Mean value and peak value

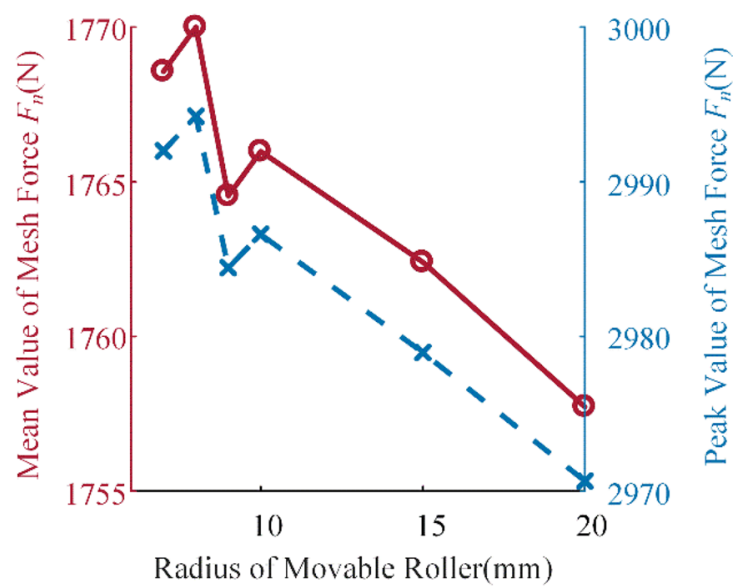

(d) Mean value and peak value

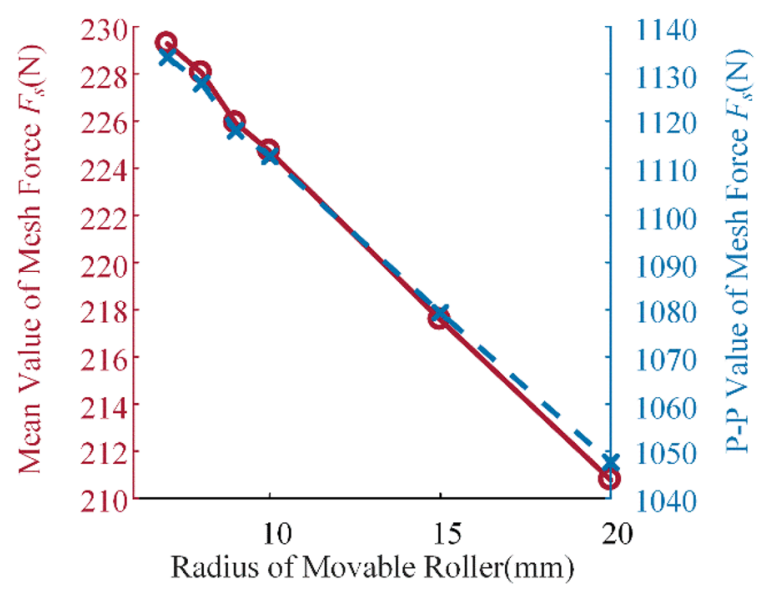

(f) Mean value and peak-peak value

Figure 12 Impacts of radius of movable roller on mesh force 


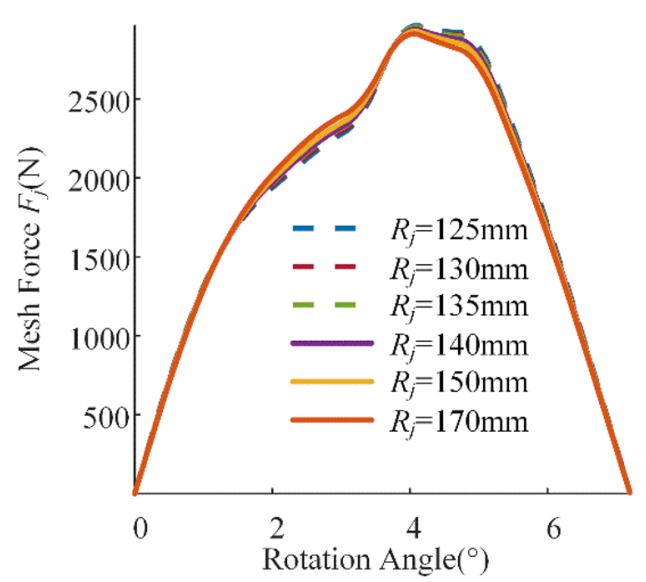

(a) Angle domain diagram

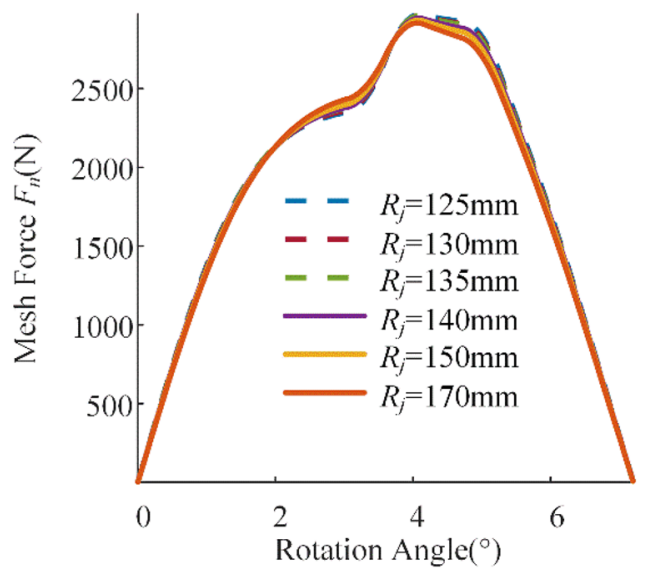

(c) Angle domain diagram

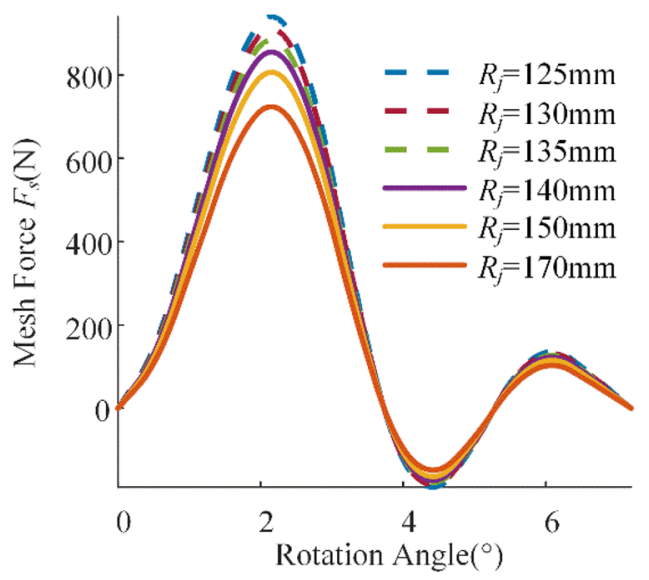

(e) Angle domain diagram

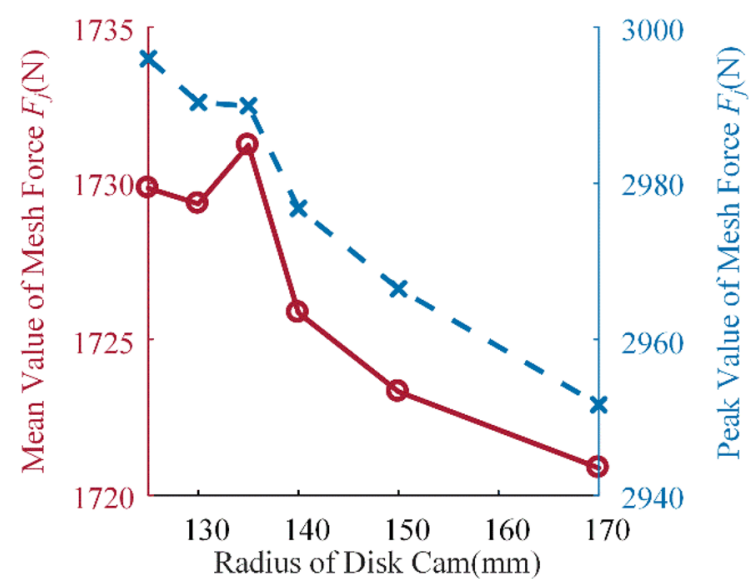

(b) Mean value and peak value

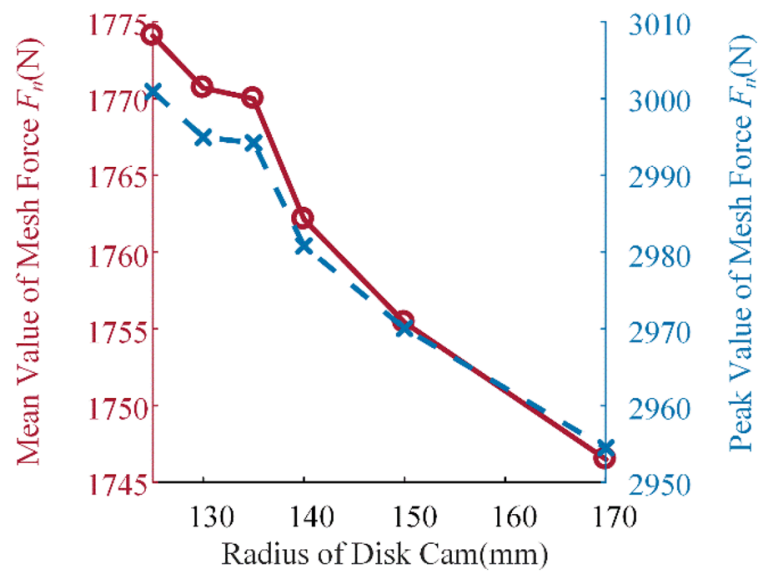

(d) Mean value and peak value

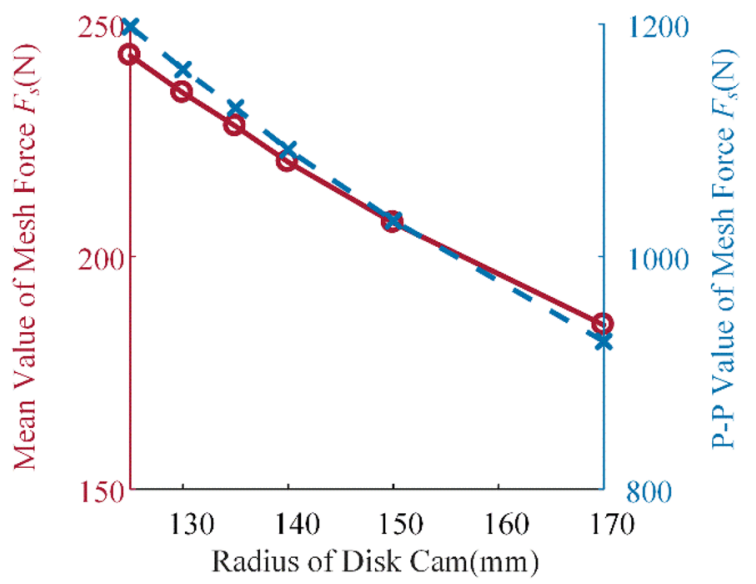

(f) Mean value and peak-peak value

Figure 13 Impacts of radius of disk cam on mesh force 


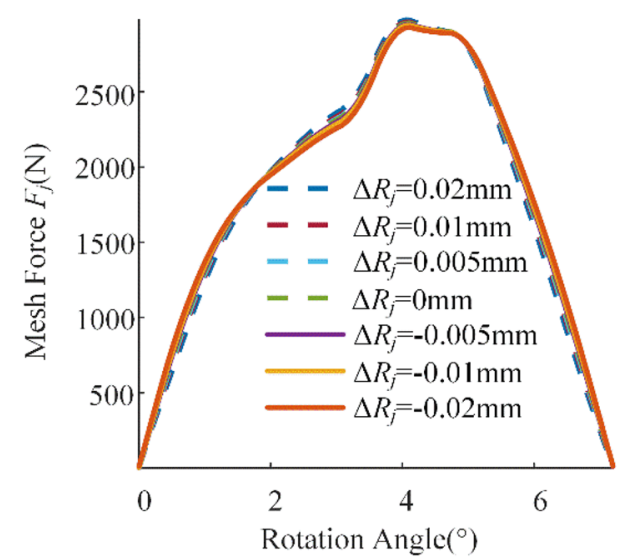

(a) Angle domain diagram

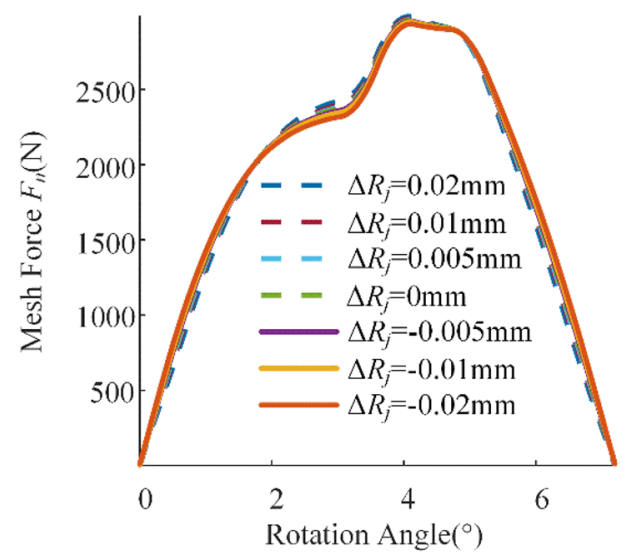

(c) Angle domain diagram

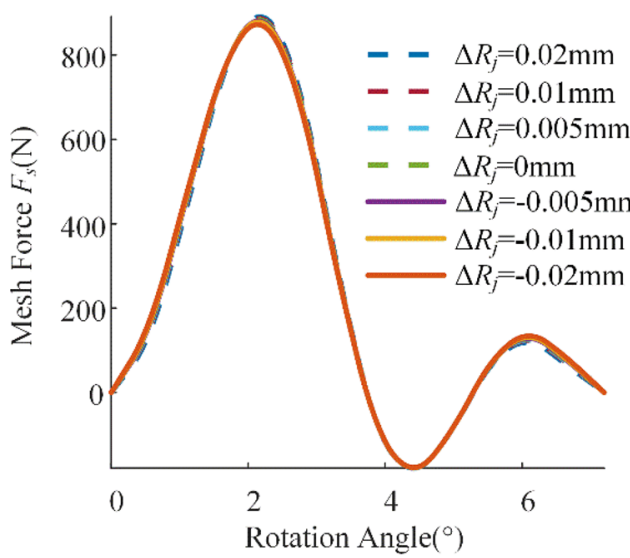

(e) Angle domain diagram

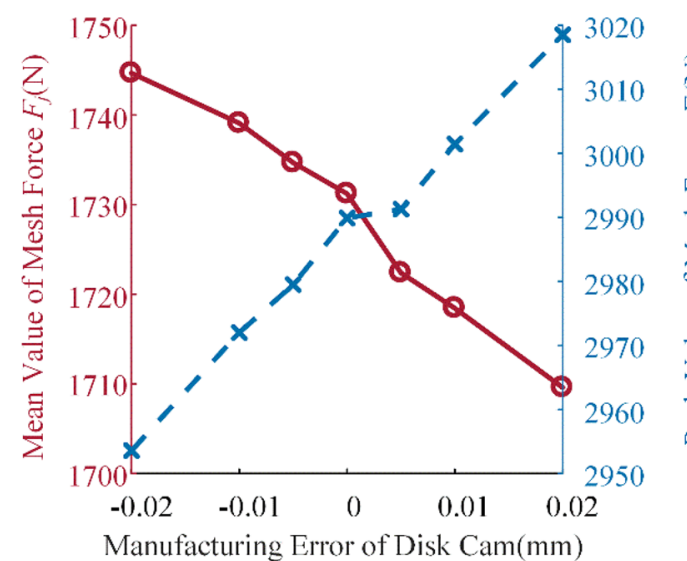

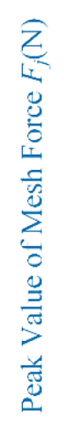

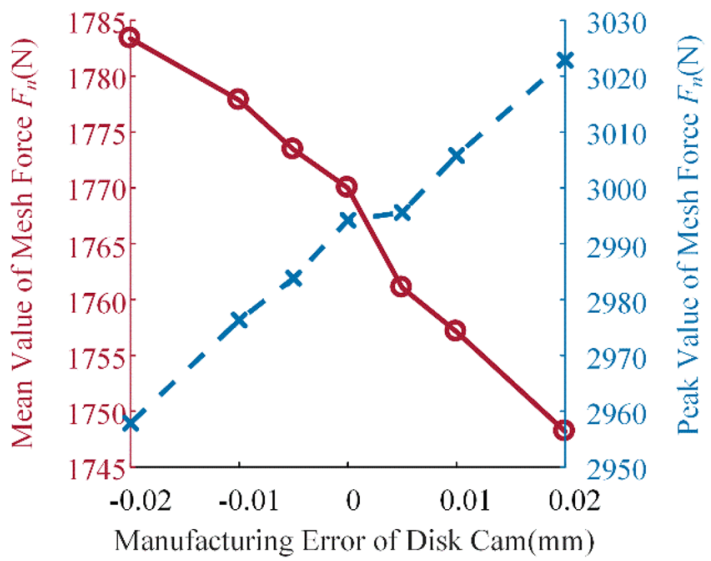

(d) Mean value and peak value

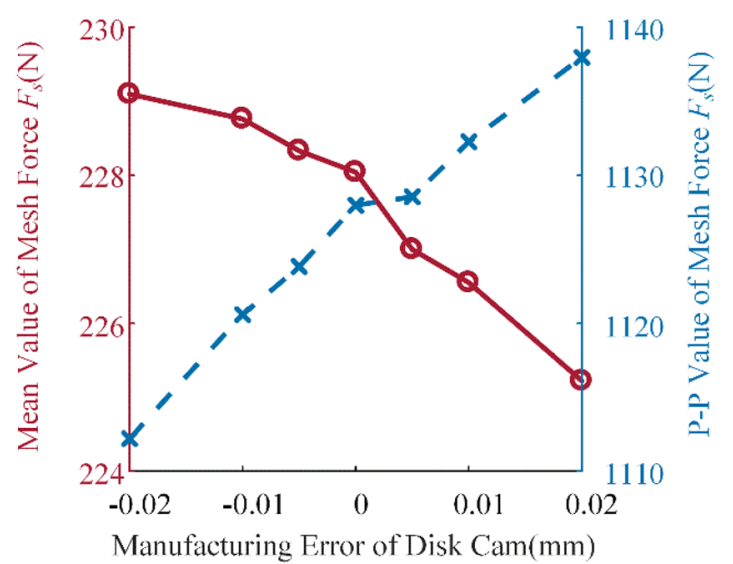

(f) Mean value and peak-peak value

Figure 14 Impacts of manufacturing error of disk cam on mesh force 


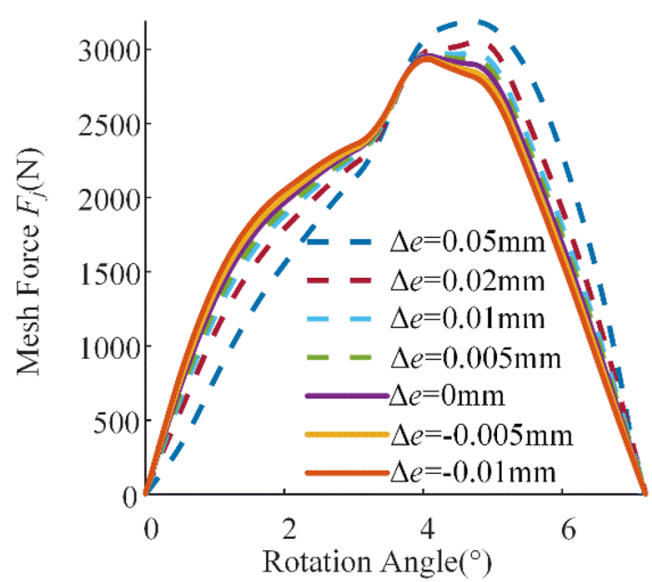

(a) Angle domain diagram

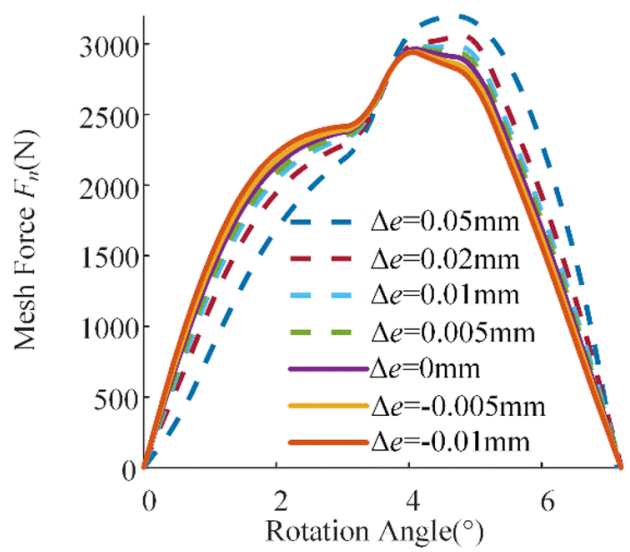

(c) Angle domain diagram

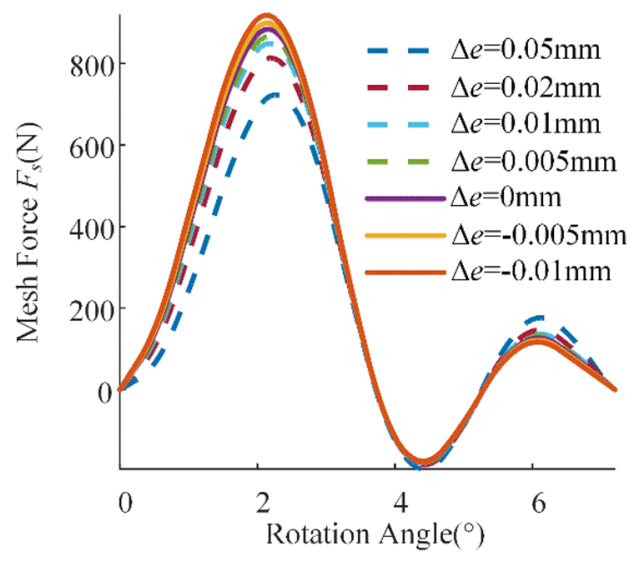

(e) Angle domain diagram

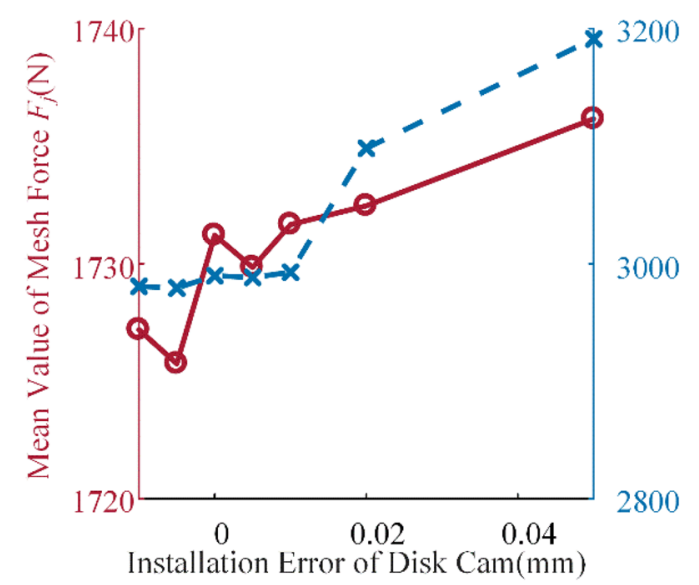

2
0
0
0
0
0
0
0
0
0
0
0
$\frac{0}{0}$
$\frac{0}{0}$
0

(b) Mean value and peak value

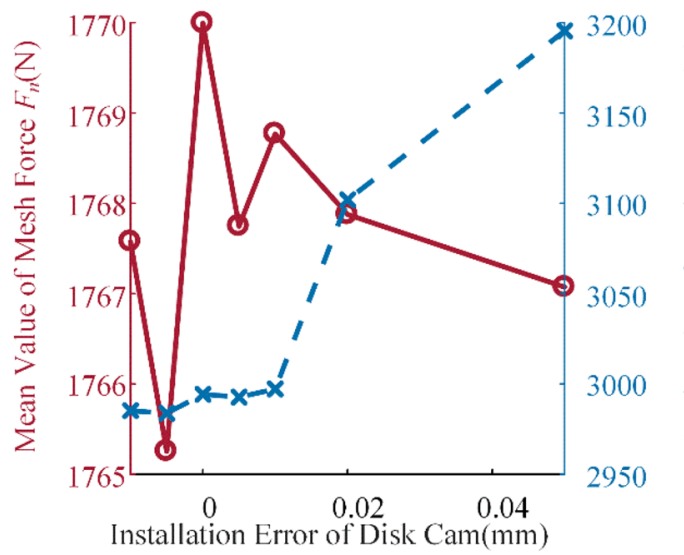

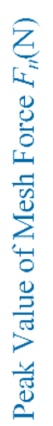

(d) Mean value and peak value

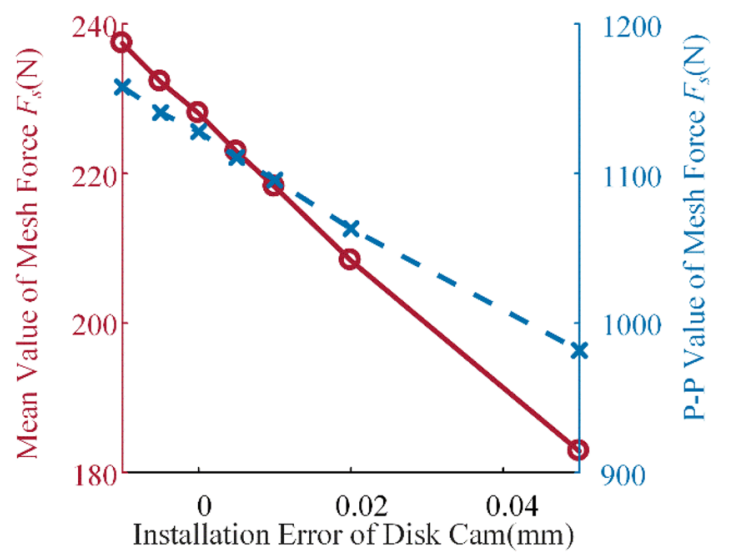

(f) Mean value and peak-peak value

Figure 15 Impacts of installation error of disk cam on mesh force 


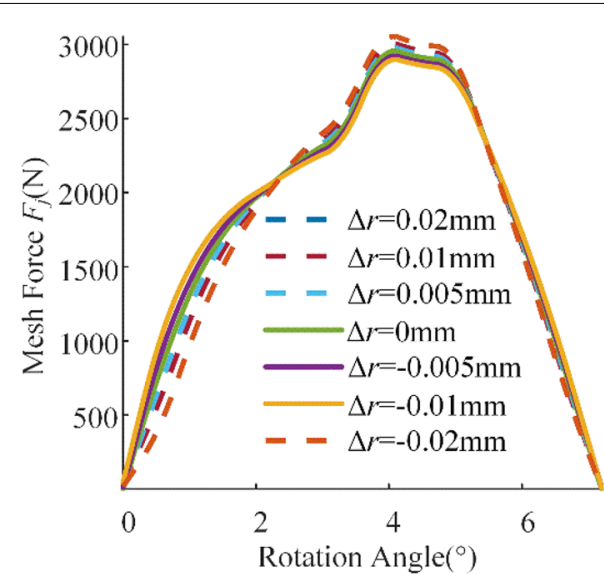

(a) Angle domain diagram

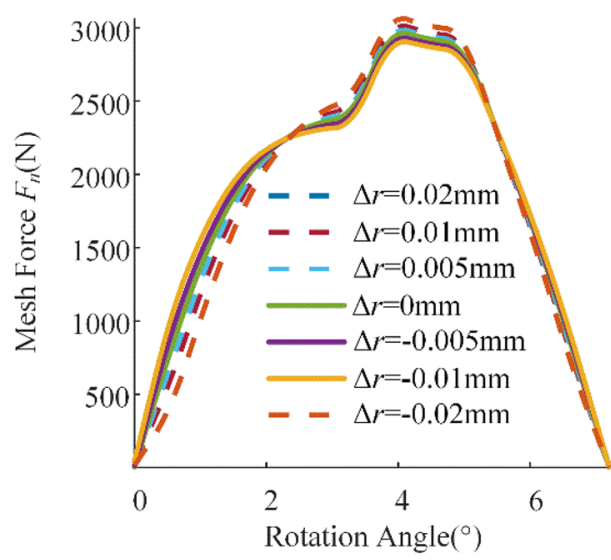

(c) Angle domain diagram

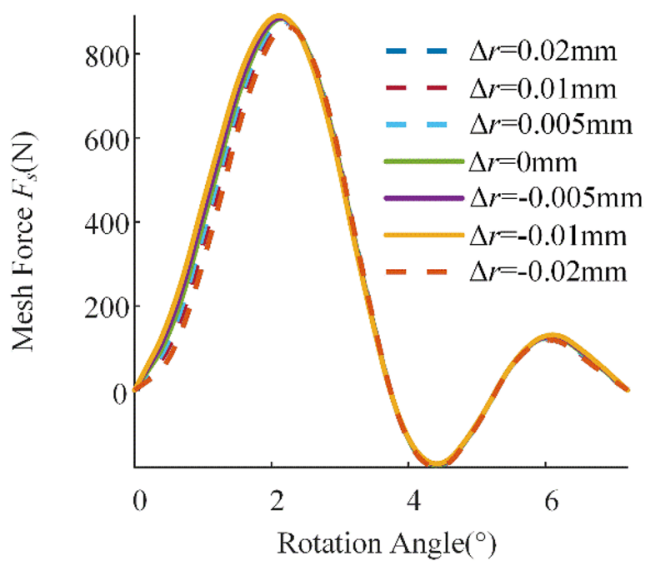

(e) Angle domain diagram

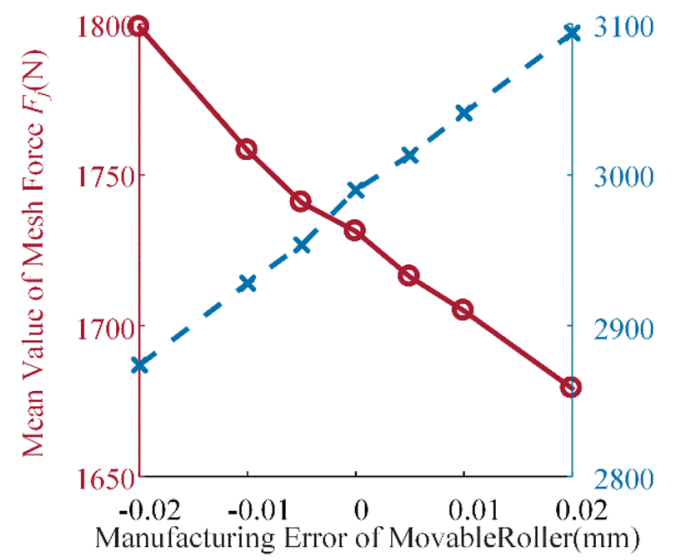

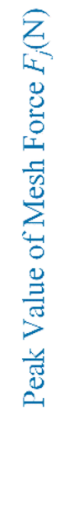

(b) Mean value and peak value

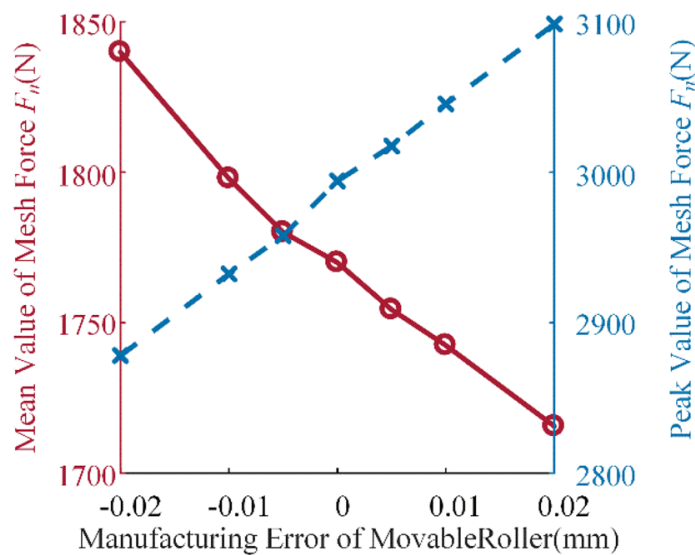

(d) Mean value and peak value

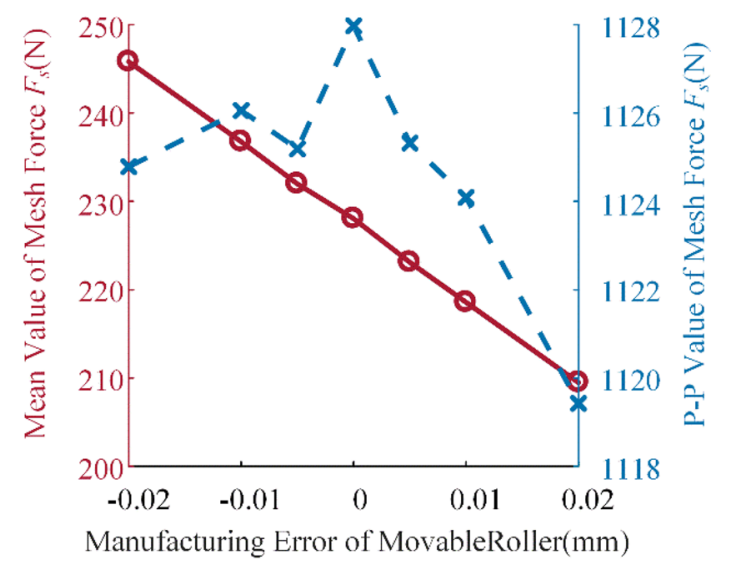

(f) Mean value and peak-peak value

Figure 16 Impacts of manufacturing error of movable roller on mesh force 
both increase by $7.7 \%$ (from $2860 \mathrm{~N}$ to $3100 \mathrm{~N}$ ). The peak to peak value of mesh force $F_{s}$ has a fluctuating change but changes a little, which means the mesh force $F_{s}$ is smoother when the movable roller has manufacturing error.

\section{Prototype and Experiment}

This section introduces the prototype of CORR and the temperature rise test, in order to prove that the design of the reducer is basically feasible. The prototype of the reducer is manufactured as shown in Figure 17 with the parameters of Table 1.

The case of the reducer is fixed in the flange which is mounted on the testing bench as shown in Figure 18. The reducer is driven by the servo motor to rotate, and it runs without load. The measurement point of temperature is on the input pinion.

The input speed gradually increases from 0 to $2000 \mathrm{r} /$ min. At the measurement point of temperature, the temperature is measured by infrared thermometer, which is $50 \mathrm{~mm}$ from the surface of the case. Record the temperature of the measurement point every $10 \mathrm{~min}$ in the noload condition. The curve of temperature rise is drawn as shown in Figure 19.

From Figure 19, we can see that the temperature becomes stable as the temperature rise gradually decreases. The experimental research results show that the temperature rise performance of the reducer meets the standard.

\section{Conclusions}

In this paper, considering the installation errors and manufacturing errors, the analytical model of the meshing force is established. Parametric studies considering various errors on the mesh force are conducted. The

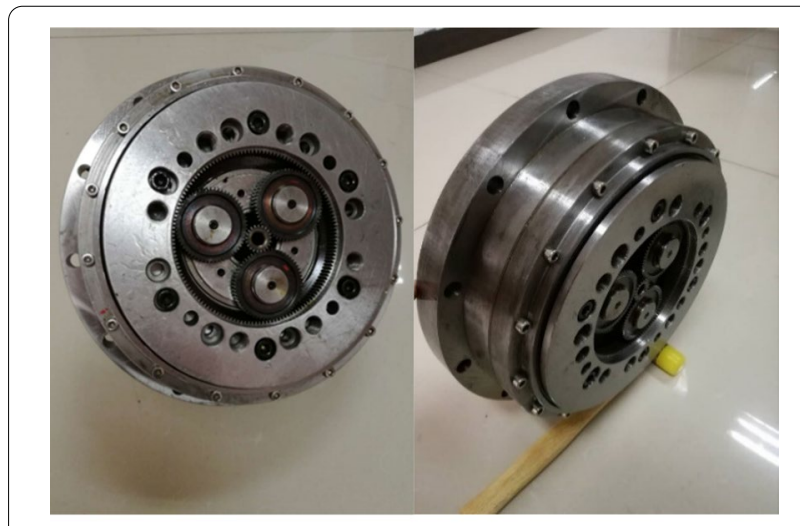

(a) Front view

(b) Oblique view

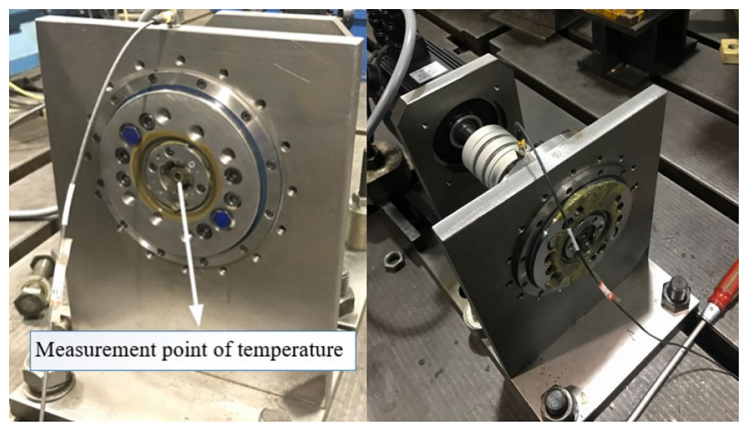

(a) Front view

(b) Oblique view

influences of manufacturing errors and installation errors on mesh force were studied. The conclusions are given as follows:

(1) To consider the manufacturing error and installation error, a mesh force modelling method with these errors was introduced in the oscillatory roller transmission. The mesh force was analyzed of movable roller by considering the manufacturing error of disk cam, installation error of disk cam and manufacturing error.

(2) As the eccentricity of disk cam increases from $2.5 \mathrm{~mm}$ to $4 \mathrm{~mm}$, the mean value of $F_{j}$ and $F_{n}$ is reduced by $17 \%$, and the mean value of $F_{s}$ has a little change. The peak value of $F_{j}$ and $F_{n}$ decrease by $16.7 \%$, and the peak to peak value of $F_{s}$ changes a little. The radius of the movable roller and the radius of the disk cam have little influence on the mesh force $F_{j}$ and $F_{n}$ but have a huge influence on $F_{s}$. When the radius of the movable roller increases from $7 \mathrm{~mm}$ to $20 \mathrm{~mm}$, the mean value of $F_{s}$ is reduced by $8 \%$, and the peak to peak

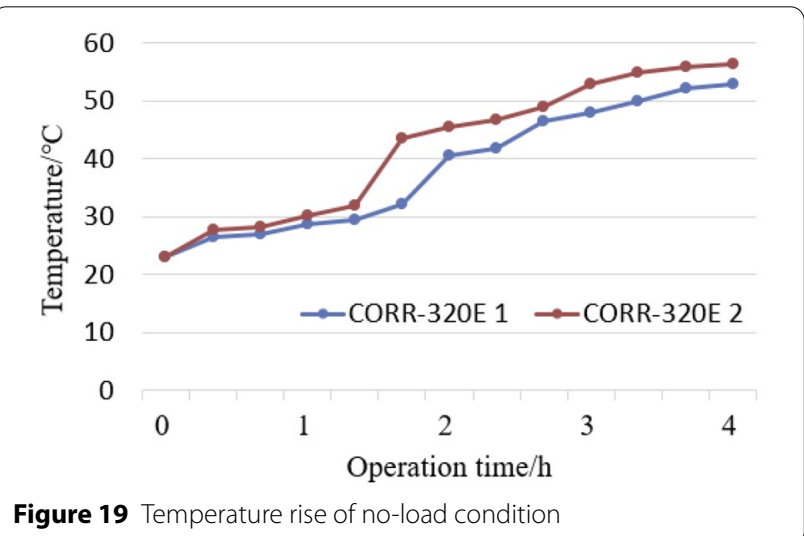

Figure 17 Prototype of the reducer 
value decreases by $8 \%$. As the radius of disk cam increases from $125 \mathrm{~mm}$ to $170 \mathrm{~mm}$, the mean value is reduced by $26.5 \%$, and the peak to peak value decreases by $22.5 \%$. As the eccentricity of disk cam, the radius of the movable roller and the radius of the disk cam increase, the tooth shape of the inner gear is more smoothly. They show that the mesh force is less when the tooth shape of the inner gear is more smoothly.

(3) The manufacturing error of disk cam has a small influence on mesh force. As the manufacturing error of disk cam grows from $-0.02 \mathrm{~mm}$ to $0.02 \mathrm{~mm}$, the mean value of the mesh force $F_{j}, F_{n}$ and $F_{s}$ all are reduced by approximate $2 \%$, and the peak value and the peak to peak value of $F_{j}, F_{n}$ and $F_{s}$ all increase by approximate $2 \%$. When the installation error of disk cam increases from $-0.01 \mathrm{~mm}$ to $0.05 \mathrm{~mm}$, the mean value of mesh force $F_{j}$ and $F_{n}$ have a fluctuating but not a huge change, and the mean value of $F_{s}$ is reduced by $25 \%$. However, the peak value of $F_{j}$ and $F_{n}$ rise smoothly as the installation error of disk cam grows to $0.01 \mathrm{~mm}$ and rises rapidly after $0.01 \mathrm{~mm}$. The peak to peak value of $F_{s}$ decreases by $14.7 \%$. As the manufacturing error of the movable roller grows from $-0.02 \mathrm{~mm}$ to $0.02 \mathrm{~mm}$, the mean value of $F_{j}$ and $F_{n}$ reduce by $6.7 \%$ and $6.5 \%$, and the mean value of $F_{s}$ decreases by $14.6 \%$. The peak value of $F_{j}$ and $F_{n}$ both increase by $7.7 \%$, and peak to peak value of $F_{s}$ has a fluctuating change but changes a little.

\section{Acknowledgements}

\section{Not applicable.}

\section{Authors' Contributions}

CW and CS were in charge of the whole research; CZ analyzed the transmission principle; $\mathrm{CL}$ and $\mathrm{LH}$ were in charge of the prototype manufacturing and experiment. All authors read and approved the final manuscript.

\section{Authors' Information \\ Changxu Wei, born in 1995, is currently pursuing the M.S. degree in mechani- cal design and theory with Chongaing University, China. His research interests} include the precision gear transmission and tooth contact analysis.

Chaosheng Song, born in 1983, is currently an associate professor at Chongqing University, China. His main research interests include gear geometry design and tooth contact analysis.

Caichao Zhu, born in 1966, is currently a professor at Chongqing University, China. His research interests include the dynamics of gear systems, the tribology of mechanical transmissions, and the design of accurate transmission.

Chengcheng Liang, born in 1993, is currently pursuing the Ph.D. degree in mechanical design and theory with Chongqing University, China. His research interests include the precision gear transmission and design of the complex gear tooth surface for spatial gear transmission.
Long Hu, born in 1995, is currently pursuing the M.S. degree in mechanical design and theory with Chongaing University, China. His research interests include the precision gear transmission and tooth contact analysis.

\section{Funding}

Supported by Research and Development Plans in Key Areas of Guangdong (Grant No. 2019B090917002) and Key Research and Development Project of Chongqing Science and Technology Program (Grant No. cstc2018jszX-cyztzxX0038).

\section{Competing Interests}

The authors declare no competing financial interests.

Received: 3 January 2020 Revised: 7 August 2021 Accepted: 20 August 2021

Published online: 03 September 2021

\section{References}

[1] J Wei, A Q Zhang, L Shi, et al. Modeling and dynamic characteristics of planetary gear transmission in non-inertial system of aerospace environment. Journal of Mechanical Design, 2019, 142(3): 1-16.

[2] Y Qin, J Q Zou, B P Tang, et al. Transient feature extraction by the improved orthogonal matching pursuit and K-SVD algorithm with adaptive transient dictionary. IEEE Transactions on Industrial Informatics, 2019: 99.

[3] S Y Liu, C S Song, C C Zhu, et al. Investigation on the influence of work holding equipment errors on contact characteristics of face-hobbed hypoid gear. Mechanism and Machine Theory, 2019, 138: 95-111.

[4] Z G Chen, W M Zhai, KY Wang. Vibration feature evolution of locomotive with tooth root crack propagation of gear transmission system. Mechanical Systems and Signal Processing, 2019, 115: 29-44.

[5] SMo, T Zhang. Analytical investigation on load sharing characteristics of herringbone planetary gear train with flexible support and floating sun gear. Mechanism and Machine Theory, 2020, 144(2): 1-27.

[6] V Sahoo, R Maiti. Load sharing by tooth pairs in involute toothed harmonic drive with conventional wave generator cam. Meccanica, 2017 53(1): 1-22.

[7] MY S Member, M I S Member, H H S Member, et al. Modeling and compensation for angular transmission error in harmonic drive gearings. IEEJ Transactions on Electrical and Electronic Engineering, 2010, 4(2): 158-165.

[8] XX Chen, Y S Liu, J Z Xing, et al. The parametric design of double-circulararc tooth profile and its influence on the functional backlash of harmonic drive. Mechanism and Machine Theory, 2014, 73: 1-24.

[9] R Dhaouadi, F H Ghorbel. Modelling and analysis of nonlinear stiffness, hysteresis and friction in harmonic drive gears. International Journal of Modelling and Simulation, 2008, 28(3): 329-336.

[10] XX Chen, S Z Lin, J Z Xing. Modeling of flexspline and contact analyses of harmonic drive. Key Engineering Materials, 2009, 419-420: 597-600.

[11] W D He, Z B Jiang, J H Bao, et al. Crank shaft modal analysis of RV reducer. Journal of Dalian Jiaotong University, 2011, 32(2): 28-32. (in Chinese)

[12] Y H Zhang, XY Wang, W D He, et al. The precise measurement technology for manufacturing error of cycloidal gear used in RV reducer. Applied Mechanics and Materials, 2017, 868: 334-339.

[13] HYu, G Liu, Y Wang, et al. The impact of gap on meshing force in twostage cycloidal gear drive. 2017 IEEE International Conference on Information and Automation (ICIA), 18-20 July 2017, Macao, China: IEEE, 2017: 760-763.

[14] XX Sun, L Han, K Ma, et al. Lost motion analysis of CBR reducer. Mechanism and Machine Theory, 2018, 120:89-106.

[15] J F Wu, X C Zhu, W Gao, et al. The dynamics analysis of rolling movable teeth reducer. Advanced Materials Research, 2011, 221:429-435.

[16] Y F Wang, L N Liu, B Yang. Virtual prototype modeling and dynamical simulation of swing movable teeth reducer. Advanced Materials Research, 2011: 531-534 
[17] S M Liang, W Luo, J G Xu, et al. Modal analysis of swing movable teeth reducer by using finite element method. Journal of Sichuan University (Engineering Science Edition), 2004, 36(2): 77-80. (in Chinese)

[18] Y Song, Q Liao, S Wei, et al. A novel approach to design a pure rolling cycloid reducer with involute teeth superimposed. 2012 Proceedings of International Conference on Modelling, Identification and Control, 24-26 June 2012, Wuhan, China: IEEE, 2012: 998-1003.

[19] J F Li, L Y Zhou, X R Dong. The influence of configuration parameters on contact ratio and teeth profile character of cam shock wave rolling movable teeth transmission. Journal of Beijing University of Technology, 2009, 35(8): 1021-1020. (in Chinese)

[20] Y L Yi, Z J An. Research on digital design and manufacture technology of rolling swing movable teeth transmission. Applied Mechanics and Materials, 2011, 86: 370-373.

[21] B Routh. Design aspects of harmonic drive gear and performance improvement of its by problems identification: A review. AlP conference proceedings, 2018: 020016

[22] A D Pham, H J Ahn. High precision reducers for industrial robots driving 4th industrial revolution: state of arts, analysis, design, performance evaluation and perspective. International Journal of Precision Engineering and Manufacturing-Green Technology, 2018, 5(4): 519-533.

[23] P L Garcia, S Crispel, E Saerens, et al. Compact Gearboxes for Modern Robotics: A Review. Frontiers in Robotics and Al, 2020, 7: 103.

[24] X Z Li, C C Song, Y Yang, et al. Optimal design of wave generator profile for harmonic gear drive using support function. Mechanism and machine theory, 2020, 152: 103941.
[25] G D Jiang, S Wang, X S M, et al. Bidirectional conjugate design method of double-circular-arc tooth profile of harmonic drives. Journal of Xi'an Jiaotong University, 2019, 53(8): 8-14. (in Chinese)

[26] Y B He, S M Liang, Y Yang. Contact mechanics of two-tooth-difference oscillating-disc movable teeth transmission. Journal of Central South University (Science and Technology), 2020, 51(1): 49-58.

[27] J A Yang, L Z Xu. Efficiency calculation and test for a two-step sine movable tooth transmission. China Mechanical Engineering, 2019, 30(21): 2577-2582, 2589. (in Chinese)

[28] F L Cao, X F Shi, L Z Xu. Stress analysis of micro movable teeth transmission systems considering van der Waals forces. China Mechanical Engineering, 2019, 30(7): 831-839. (in Chinese)

[29] J F Li, H W Zhao, L Y Zhang, et al. Transmission and tooth profile equation of swing output movable teeth cam mechanism. Journal of Mechanical Engineering, 2018, 54(23): 23-31. (in Chinese)

[30] Y L Yi, P P Liu, Z J An, et al. Tooth profile design and meshing characteristics analysis of any tooth-difference pure rolling movable tooth transmission. Journal of Mechanical Engineering, 2016, 52(11): 50-56. (in Chinese)

[31] J F Li, X R Dong. Method of meshing force analysis for the movable rolling teeth transmission with cam actuating. Journal of Mechanical Engineering, 2008(05): 39-44. (in Chinese)

\section{Submit your manuscript to a SpringerOpen ${ }^{\circ}$ journal and benefit from:}

- Convenient online submission

- Rigorous peer review

- Open access: articles freely available online

- High visibility within the field

- Retaining the copyright to your article

Submit your next manuscript at $\boldsymbol{\nabla}$ springeropen.com 